\title{
Pharmacological Properties of
} Chalcones: A Review of Preclinical Including Molecular Mechanisms and Clinical Evidence

\section{OPEN ACCESS}

Edited by:

Andrei Mocan,

Iuliu Haţieganu University of Medicine and Pharmacy, Romania

Reviewed by: Iulia Popescu, University of Pittsburgh, United States

Priyia Pusparajah, Monash University Malaysia, Malaysia

*Correspondence:

Javad Sharifi-Rad javad.sharifirad@gmail.com Abdelhakim Bouyahya boyahyaa-90@hotmail.fr

Constantin Caruntu costin.caruntu@gmail.com Abhiijt Dey abhijit.dbs@presiuniv.ac.in Daniela Calina calinadaniela@gmail.com

Specialty section: This article was submitted to Ethnopharmacology,

a section of the journal Frontiers in Pharmacology

Received: 07 August 2020 Accepted: 12 November 2020 Published: 18 January 2021

Citation:

Salehi B, Quispe C, Chamkhi I, El Omari N, Balahbib A, Sharifi-Rad J,

Bouyahya A, Akram M, lqbal M,

Docea $A O$, Caruntu $C$, Leyva-Gómez G, Dey A, Martorell M, Calina D, López V and Les F (2021)

Pharmacological Properties of Chalcones: A Review of Preclinical Including Molecular Mechanisms and

Clinical Evidence.

Front. Pharmacol. 11:592654. doi: 10.3389/fphar.2020.592654
Bahare Salehi ${ }^{1}$, Cristina Quispe ${ }^{2}$, Imane Chamkhi ${ }^{3,4}$, Nasreddine El Omari ${ }^{5}$, Abdelaali Balahbib ${ }^{6}$, Javad Sharifi-Rad ${ }^{7,8 *}$, Abdelhakim Bouyahya ${ }^{9 *}$, Muhammad Akram ${ }^{10}$, Mehwish lqbal ${ }^{11}$, Anca Oana Docea ${ }^{12}$, Constantin Caruntu ${ }^{13,14 *}$, Gerardo Leyva-Gómez ${ }^{15}$, Abhijit Dey ${ }^{16 *}$, Miquel Martorell ${ }^{17,18}$, Daniela Calina ${ }^{19 *}$, Víctor López ${ }^{20,21}$ and Francisco Les ${ }^{20,21}$

${ }^{1}$ Medical Ethics and Law Research Center, Shahid Beheshti University of Medical Sciences, Tehran, Iran, ${ }^{2}$ Facultad de Ciencias de La Salud, Universidad Arturo Prat, lquique, Chile, ${ }^{3}$ Faculty of Sciences, Mohammed V University of Rabat, Rabat, Morocco, ${ }^{4}$ Laboratory of Plant-Microbe Interactions, AgroBioSciences, Mohammed VI Polytechnic University, Ben Guerir, Morocco, ${ }^{5}$ Laboratory of Histology, Embryology, and Cytogenetic, Faculty of Medicine and Pharmacy, Mohammed V University in Rabat, Rabat, Morocco, ${ }^{6}$ Laboratory of Zoology and General Biology, Faculty of Sciences, Mohammed V University in Rabat, Rabat, Morocco, ${ }^{7}$ Phytochemistry Research Center, Shahid Beheshti University of Medical Sciences, Tehran, Iran, ${ }^{8}$ Facultad de Medicina, Universidad del Azuay, Cuenca, Ecuador, ${ }^{9}$ Laboratory of Human Pathologies Biology, Department of Biology, Faculty of Sciences, and Genomic Center of Human Pathologies, Faculty of Medicine and Pharmacy, Mohammed V University Rabat, Rabat, Morocco, ${ }^{10}$ Department of Eastern Medicine, Government College University, Faisalabad, Pakistan, ${ }^{11}$ Institute of Health Management, Dow University of Health Sciences, Karachi, Pakistan, ${ }^{12}$ Department of Toxicology, University of Medicine and Pharmacy of Craiova, Craiova, Romania, ${ }^{13}$ Department of Physiology, "Carol Davila" University of Medicine and Pharmacy, Bucharest, Romania, ${ }^{14}$ Department of Dermatology, "Prof. N.C. Paulescu" National Institute of Diabetes, Nutrition, and Metabolic Diseases, Bucharest, Romania, ${ }^{15}$ Departamento De Farmacia, Facultad De Química, Universidad Nacional Autónoma De México, Ciudad De México, Mexico, ${ }^{16}$ Department of Life Sciences, Presidency University, Kolkata, India, ${ }^{17}$ Department of Nutrition and Dietetics, Faculty of Pharmacy, and Centre for Healthy Living, University of Concepción, Concepción, Chile, ${ }^{18}$ Unidad De Desarrollo Tecnológico, UDT, Universidad De Concepción, Concepción, Chile, ${ }^{19}$ Department of Clinical Pharmacy, University of Medicine and Pharmacy of Craiova, Craiova, Romania, ${ }^{20}$ Department of Pharmacy, Faculty of Health Sciences, Universidad San Jorge, Zaragoza, Spain, ${ }^{21}$ Instituto Agroalimentario De Aragón-IA2 CITA-Universidad De Zaragoza, Zaragoza, Spain

Chalcones are among the leading bioactive flavonoids with a therapeutic potential implicated to an array of bioactivities investigated by a series of preclinical and clinical studies. In this article, different scientific databases were searched to retrieve studies depicting the biological activities of chalcones and their derivatives. This review comprehensively describes preclinical studies on chalcones and their derivatives describing their immense significance as antidiabetic, anticancer, anti-inflammatory, antimicrobial, antioxidant, antiparasitic, psychoactive, and neuroprotective agents. Besides, clinical trials revealed their use in the treatment of chronic venous insufficiency, skin conditions, and cancer. Bioavailability studies on chalcones and derivatives indicate possible hindrance and improvement in relation to its nutraceutical and pharmaceutical applications. Multifaceted and complex underlying mechanisms of chalcone actions demonstrated their ability to modulate a number of cancer cell lines, to inhibit a number of pathological microorganisms and parasites, and to control a number of signaling molecules and cascades related to disease modification. Clinical studies on chalcones revealed general absence of adverse effects besides reducing the clinical signs and symptoms with decent bioavailability. Further studies are needed to elucidate their 
structure activity, toxicity concerns, cellular basis of mode of action, and interactions with other molecules.

Keywords: chalcones, flavonoids, bioavailability, pharmacological studies, molecular mechanisms, clinical trials

\section{INTRODUCTION}

Chalcones are among the leading categories of flavonoids across the entire kingdom of plant (Hideo and Tatsurou, 1997; Abbas et al., 2014). The term chalcone is originated from the Greek name chalcos which means bronze. Chalcones were initially manufactured in the research lab in late 1800s (Shimokoriyama, 1962). The chalcone chemistry has created thorough scientific research all the way through the globe (Hideo and Tatsurou, 1997).

Naturally existing chalcones were not separated till the year 1910 (Shimokoriyama, 1962). Chalcones that derived from nature exist mostly as colors of petal and furthermore have been established in the heartwood, leaf, bark, fruit, and root of a range of plants and botanicals (Schroder, 1999).

Chalcones are also recognized as benzyl acetophenone. Chalcones are alpha, beta unsaturated ketones holding two fragrant rings (rings $\mathrm{A}$ and $\mathrm{B}$ ) having different arrangement of substituents. In chalcones, two fragrant rings are connected by an aliphatic three carbon series (Rojas et al., 2002) (Figure 1).

Plants containing chalcones, for instance, the Glycyrrhiza, Piper, Angelica, and Ruscus genus, have long been utilized as therapeutic remedies in Balkan countries (Schroder, 1999; Chatzopoulou et al., 2013; Maccari and Ottana, 2015). Numerous unadulterated chalcones were accepted for clinical applications or experimented in humans. Licochalcones segregated from the plant of licorice has been stated to have a range of biological activities, for instance, antispasmodic, chemopreventive, antimalarial, antitumour, anti-inflammatory, antifungal, antioxidant, and antibacterial activities (Real, 1967; Takahashi et al., 1998). Both apples and sour fruits are loaded nutritional sources of dihydrochalcones and chalcones. Moreover, these complexes could even compose a better contribution to the overall daily consumption of unrefined or organic polyphenolics compounds than other considerably researched flavonoids (Tomás-Barberán and Clifford, 2000).

The purpose of this review is to summarize the most important pharmacological activities highlighting the cellular and molecular mechanisms of action of natural and synthetic chalcones, to better understand their therapeutic potential in the future.

\section{METHODOLOGY}

\section{Search Strategy}

An extensive research was conducted into the available scientific databases PubMed, Scopus, Scielo, and Science Direct using the terms "chalcones," "bioavailability," "biological activities," "antiinflammatory," "antidiabetic," "neuroprotective," "antioxidant," "anticancer," "antibacterial," and "antifungal."

\section{Inclusion Criteria}

The inclusion criteria included research studies or reviews that reported the pharmacological actions of chalcones were included; articles published in English, book chapters that also included phytochemical data, and preclinical studies on cell cultures or animal model with evidence of cellular and molecular mechanisms of action; studies that included chalcones and their derivatives from plants whose nomenclature is included in the Plant List (http://www.theplantlist.org/).

\section{Exclusion Criteria}

The exclusion criteria included abstracts, case reports, and conference proceedings that did not meet the inclusion criteria, as well as studies that included homeopathic preparations.

\section{Data Collection}

Selected pharmacological studies included data on chalcones and their derivatives analyzed, experimental model (in vivo or in vitro), dose, concentration, and results of pharmacological activities with molecular mechanisms included. All information obtained and analyzed in this comprehensive and updated review were summarized in tables and figures.

\section{PRECLINICAL PHARMACOLOGICAL ACTIVITIES OF CHALCONES}

Preclinical studies on chalcones and their derivatives have shown their high potential as antidiabetic, anticancer, antiinflammatory, antimicrobial, antioxidant, antiparasitic, psychoactive, and neuroprotective agents (Figure 2).

\section{Antidiabetic Activity}

\section{In Vitro Antidiabetic Activity}

Several synthetic chalcones have been reported to have potential inhibitory activity against $\alpha$-glucosidase or $\alpha$-amylase.

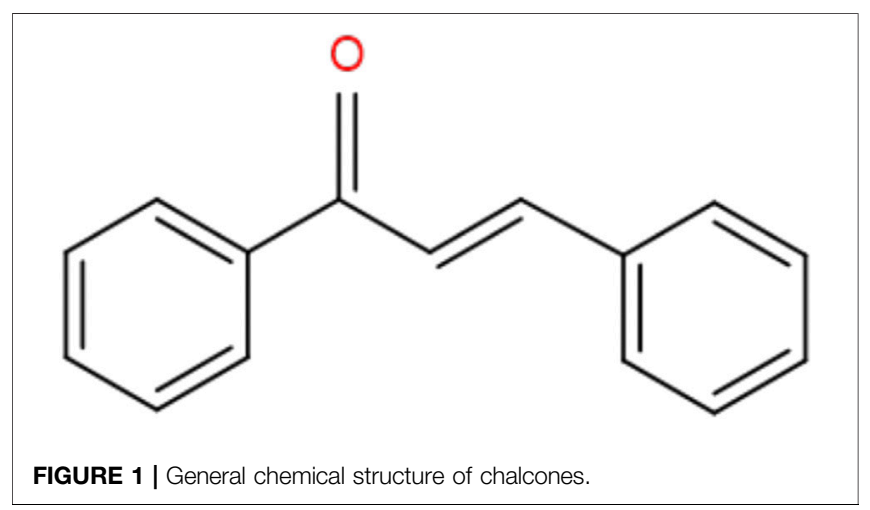




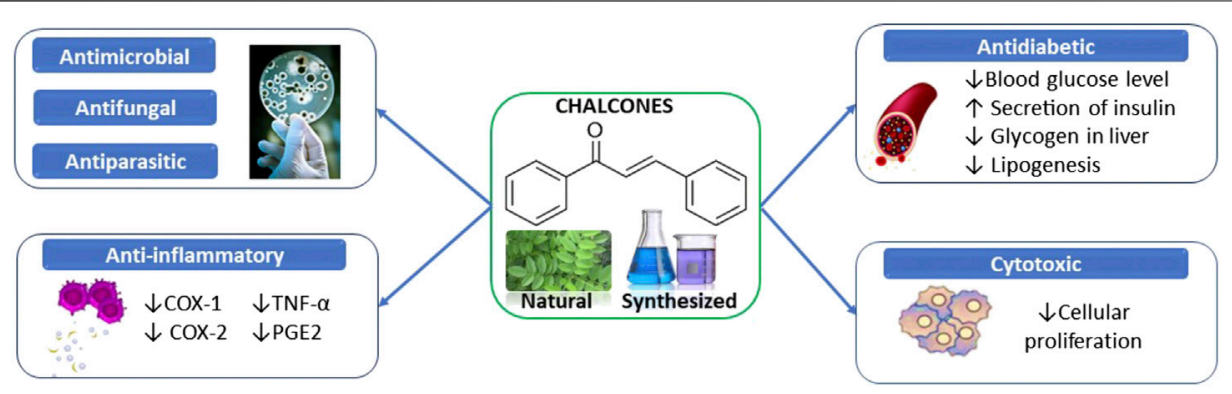

FIGURE 2 | Summarized scheme of the most important pharmacological properties of chalcones.

The $\mathrm{IC}_{50}$ value of synthetic intermediate chalcones (1-24) varied between $15 \pm 0.14$ and $385 \pm 5.60 \mu \mathrm{M}$ (Ansari et al., 2005). Similar observations were noted with the Chana series (Bak et al., 2011), and with the tris-chalcone derivatives (5a-5i), all showing higher inhibition profiles than those of acarbose (Burmaoglu et al., 2019). Studies on hydroxyl chalcones and bis-chalcones (1a-1m) and (2a$2 \mathrm{~m}$ ) were performed in connection to the inhibition kinetics (Cai et al., 2017). By using the abovementioned methods, natural chalcone derivatives (morusalbins A-D) showed significant inhibitory activities against a-glucosidase (Ha et al., 2018). 3',5'-digeranylated chalcone (16) demonstrated noncompetitive inhibition characteristics (Ryu et al., 2010; Sun et al., 2015). In another study, compound $4 \mathrm{~m}$ was found to be the most active compared to the other chalcone-triazole derivatives (Chinthala et al., 2015). Numerous studies have shown some chalcones and/or their derivatives (such as chalcone 1 with an $\mathrm{IC}_{50}$ of $840 \pm 2.50 \mu \mathrm{M}$ while that of acarbose was $860.23 \pm 6.10 \mu \mathrm{M}$ ) with significant inhibitory effects than those of the standards used (Imran et al., 2015; Monisha et al., 2018).

Chalcone units of conjugates also exhibited moderate inhibitory activities against $\boldsymbol{\alpha}$-glucosidase (Tang et al., 2014), with the highest activity $\left(\mathrm{IC}_{50}=3.2 \pm 0.2 \mu \mathrm{M}\right)$ recorded by conjugate $1 \mathrm{~b}$. Moreover, moderate inhibitory effect was observed by piperonal chalcones derivatives against $\boldsymbol{\alpha}$-amylase (Acharjee et al., 2018).

Four chalcone derivatives were synthesized, and it was found that the compound 3-(4-hydroxyphenyl)-1-phenylprop-2-en-1-one has an inhibitory effect on $\alpha$-amylase (Attarde et al., 2014). Chalcone 4 (butein) has been shown to be the most potent compound among 41 derivatives, exhibiting significant inhibition of a-glucosidase, moderate inhibition of $a$-amylase, and competitive inhibition of both the enzymes (Rocha et al., 2019).

In another study, chalcone 20 was the most active inhibitor $\left(\mathrm{IC}_{50}=0.4 \mu \mathrm{M}\right)$ of $\alpha$-glucosidase among 20 derivatives, exhibiting noncompetitive inhibition (Seo et al., 2005; Tajuddeen et al., 2018). In addition, the inhibitory capacity of chalcones $1-13$ and bis-chalcones $14-18$ against $\alpha$-amylase $\left(\mathrm{IC}_{50}=1.25 \pm 1.05-2.40 \pm\right.$ $0.09 \mu \mathrm{M})$ was found to be comparable to that of acarbose $\left(\mathrm{IC}_{50}=\right.$ $1.04 \pm 0.3 \mu \mathrm{M})$ (Attarde et al., 2014). Furthermore, researchers have recorded promising activities of different chalcones in inhibiting the aforementioned enzymes, occupying the active sites (Najafian et al., 2010; Rawat et al., 2011; Gomes et al., 2017).

A study evaluated the antidiabetic activity of sulfonamide chalcone derivatives in silico using methods like homology modeled structure, molecular docking, and MD simulation. This study indicated that these derivatives can bind to residues of the active site as the same way as drugs such as acarbose and voglibose (Bharatham et al., 2008).

Prenylated chalcones $(3,4,7)$ and flavanone-coupled chalcones $(9,12,13)$ of Boesenbergia rotunda (L.) Mansf. roots exhibited inhibition greater than $90 \%$ at the concentration of $20 \mu \mathrm{g} / \mathrm{ml}$ plus an inhibitory power of $\alpha$-glucosidase higher than that of acarbose $\left(\mathrm{IC}_{50}=1.2 \mathrm{mM}\right)$ (Chatsumpun et al., 2017). A natural chalcone (lavandulylated chalcone) exhibited inhibitory activity against $\beta$-glucosidase $\left(\mathrm{IC}_{50}=57 \mu \mathrm{M}\right.$ ) while noncompetitively inhibiting a-glucosidase (Kim et al., 2006). Similarly, another study isolated xanthohumol $(\mathrm{XN})$ from Humulus lupulus L. as a potential inhibitor of $a$-glucosidase $\left(\mathrm{IC}_{50}=8.8 \mu \mathrm{M}\right)$ reversibly and noncompetitively (Liu et al., 2014). Other natural chalcones (6, $7,20)$ were identified by from Derris indica (Lam.) Bennet root extract as a moderate inhibitor of a-glucosidase, and compound 6 showed the most potent activity $\left(\mathrm{IC}_{50}=103.5 \mu \mathrm{M}\right)$ (Rawat et al., 2011).

Natural prenylchalconaringenins (1) and (2) have been investigated for their inhibitory properties against digestive enzymes; 3 '-geranylchalconaringenin (2) showed moderate inhibition of $\alpha$-amylase $\left(\mathrm{IC}_{50}=20.46 \mu \mathrm{M}\right)$ and competitive and irreversible inhibition of a-glucosidase $\left(\mathrm{IC}_{50}=1.08 \mu \mathrm{M}\right.$ ) (Sun et al., 2017). In addition, these two enzymes were also inhibited by three natural chalcones from Psoralea corylifolia (Mounika, 2015). Another chalcone $\quad\left(2^{\prime}, 4^{\prime}\right.$-dihydroxy-6'-methoxy- $3^{\prime}, 5^{\prime}$ dimethylchalcone) (DMC) from Cleistocalyx operculatus (Roxb.) Merr. and L.M.Perry flower buds inhibited pancreatic a-amylase $\left(\mathrm{IC}_{50}=69.35\right)$ (Zhang and Lu, 2012). Regarding GLUT4-dependent glucose uptake, 4-hydroxyderricin (4HD) and xanthoangelol (XAG), two natural chalcones from Angelica keiskei (Miq.) Koidz. stem juice, increased this uptake via the signaling pathway of LKB1/AMPactivated protein kinase in 3T3-L1 adipocytes (Ohta et al., 2015).

\section{In Vivo Antidiabetic Activity}

Several authors have evaluated the antihyperglycemic activity of synthetic chalcones in streptozotocin-induced diabetic rats (Satyanarayana et al., 2004; Shukla et al., 2007; Najafian et al., 2010; Rawat et al., 2011; Mahapatra et al., 2017a; Sengupta et al., 2017; Shukla et al., 2017; Tajammal et al., 2017; Acharjee et al., 2018; Naidu, 2018; Raju et al., 2018). It was found that these compounds have a moderate to potential ability to reduce blood sugar. The same effect was noted in starch-loaded rats, using 
chalcone derivative 8c (Rawat et al., 2011). Moreover, serum glucose levels were measured in hyperglycemic rats treated with chalcone analogs, which showed a significant antihyperglycemic effect (Alberton et al., 2008).

In a study conducted by Damazio et al., it was evaluated the antihyperglycemic activity of nitrochalcones (Damazio et al., 2009) and naphthylchalcones (Damazio et al., 2010) in diabetic rats by determining blood glucose levels, insulin secretion, and 14C-glucose uptake into the soleus muscle of the animal. This indicates that the effect of chalcones on lowering blood glucose in the hyperglycemic rat can be attributed mainly to insulin secretion with potency similar to that of glipizide. In addition, the glycogen levels in the liver, brain, and spinal cord of rats were estimated following $25 \mathrm{mg} / \mathrm{kg}$ dose of chalcone administration for 7 days to discover that these chalcones were able to reduce the glycogen content in the liver, and therefore exerted a strong antidiabetic activity (Jamal et al., 2009). Furthermore, when 2-hydroxychalcone was administered to male rats, they rendered insulin resistance by a high fructose diet. This chalcone was found to have significant hypoglycemic activity by increasing insulin secretion and glycosylated hemoglobin (Jayanthi et al., 2012).

Chalcone derivatives (4A-4E) were tested on sucrose-loaded diabetic albino mice to find that compound 4-C (2-(3-(4methoxyphenyl)-1H-pyrazol-5-yl) phenol) achieved the most promising activity, which is supported by docking study (Jain and Jain, 2017). For male mice (type 2 diabetes), at doses of 200-300 mg/kg/day, 2', 4'-dihydroxy-4-methoxydihydrochalcone (DMC-2) exhibited a hypoglycemic effect comparable to that of metformin (antidiabetic drug) (Ribnicky et al., 2009).

Chalcone derivatives (13a-h) and (19a-h) instreptozotocininduced diabetic mice, compounds13e, 13g, and $19 \mathrm{f}$ reduced TG, TC, and Glu levels, respectively (Zhu et al., 2018). Diabetic mice were treated with trihydroxychalcone derivatives, and therefore, chalcone 13 stimulated activation of AMP-activated protein kinase (AMPK), increased muscle FAO, improved tolerance to glucose, and decreased fat accumulation in the liver and skeletal muscles (Shin et al., 2018). Hypoglycemic activity of sulfonylurea chalcones 1-3 was also exhibited in normoglycemic rabbits to show that all these chalcones have activity comparable to that of gliclazide (Rao et al., 2014).

Significant hypoglycemic effects were displayed by five isoliquiritigenin (ISL) derivatives isolated from Glycyrrhiza glabra L. rhizomes tested in streptozotocin-induced diabetic mice (Gaur et al., 2014), chalcone-6'-hydroxy-2',3,4trimethoxy-4'-O- $\beta$-D-glucopyranoside (1) from Pouzolzia rugulosa (Wedd.) Acharya \& Kravtsova. leaves tested in alloxan-induced diabetic mice (Semwal et al., 2009), and 2'4dihydroxy chalcone-4-glucoside from Adhatoda zeylanica Medik. flower (Purnima et al., 2012). Likewise, in mice with hyperglycemia, xanthoangelol (XA) and 4-hydroxyderricin (4HD), two major types of chalcones derived from Angelica keiskei (Miq.) Koidz. lowered blood sugar by demonstrating insulin-like activity with preventive effects of (4HD) on the development of diabetes in genetically diabetic $\mathrm{KK}-\mathrm{A}^{\mathrm{y}}$ mice (Enoki et al., 2007; Enoki et al., 2010).
Table 1 summarizes the in vitro and in vivo antidiabetic properties of natural and synthetic chalcones.

\section{Anti-Inflammatory Activity}

Literature reported several chalcones and their derivative that have shown promise to inhibit cyclooxygenase (COX) (Table 2) (Araico et al., 2006; Nyandoro et al., 2012; Bano et al., 2013; Jantan et al., 2014; Özdemir et al., 2015; Okuda-Tanino et al., 2017; Farzaneh et al., 2018). In a study to assess the antiinflammatory effect, new chalcone derivatives using carrageenan-induced hind paw edema model, the results showed that $5^{\prime}$-chloro-2'-hydroxy- $4^{\prime} 6^{\prime}$-dimethyl-3, 4, 5trimethoxychalcone (1) exhibited the most potent antiinflammatory activity with a $90 \%$ inhibition of edema (Bano et al., 2013). In another study, a novel class indole-based chalcones were evaluated for their inhibitory effects on COX-1 and COX-2, and showed remarkable inhibition of COX-1 (Özdemir et al., 2015). The nitrogen-containing chalcone derivatives showed inhibition of some enzymes implicated to inflammatory process such as $\beta$-glucuronidase, COX-2, and trypsin (Bandgar et al., 2010). In another investigation, the synthetic fluoro-hydroxy substituted pyrazole chalcones demonstrated that exhibited selective inhibitory effect against COX-2 enzyme and a moderate effect against COX-1. The activity was related to the inhibition of COX-2 (Jadhav et al., 2013).

Natural chalcones have also shown their ability to inhibit COX-1 and COX-2: 2-hydroxy-3,4,6-trimethoxychalcone isolated from Toussaintia orientalis Verdc. root and stem bark extracts had a potent inhibitory effect against both the enzymes (Nyandoro et al., 2012).

Chalcones exhibited promising activity against NO and PGE2 (Table 2). The effect of dimethylamino-chalcones on the generation of NO and PGE2 mediators was studied in LPSstimulated RAW 264.7 macrophage cells. The results showed that chalcones suppressed NO production in a dose-depending manner (Rojas et al., 2002). In another study, in order to evaluate the inhibitory effects of trimethoxychalcone derivatives on $\mathrm{NO}$ production, the results showed a suppression of NO and PGE2 in LPS-activated RAW 264.7 macrophage cells by 2,4,6-trimethoxy20 -trifluoromethylchalcone. This suggestion was supported by the data which showed an inhibition of nitrite and PGE2 levels (Rojas et al., 2003a; Rojas et al., 2003b).

Natural chalcones have also shown the ability to inhibit NO and PGE2 production. Mallotophilippen chalcones isolated from Mallotus philippinensis fruit extracts, exhibited suppression of NO synthesis in a murine macrophage-like cell line (Daikonya et al., 2004). Xanthohumol and dihydroxanthohumol isolated from Humulus lupulus L. are other natural chalcones, which considerably inhibited NO production by suppressing iNOS induced by LPS and INF- $\gamma$ in a murine macrophage-like cell line (Zhao et al., 2003).

Chalcones also have proved their ability to inhibit NF- $\kappa \mathrm{B}$ (Gilmore, 2006; Mahapatra et al., 2017b; Chu and Guo, 2016). Other chalcone derivatives such as isoliquiritigenin, butein, and homobutein (Orlikova et al., 2012) have suppressed TNF- $\alpha$ mediated by the inhibition of NF- $\mathrm{B}$ gene expression 
TABLE 1 | Antidiabetic activities of chalcones: in vitro and in vivo preclinical pharmacological studies.

Chalcones/source
1-\{3-[3-(substituted phenyl) prop-2-enoyl] phenyl\}
thioureas/synthesized
Intermediate chalcones 1-24/synthesized
Chalcone derivatives (MVC1-MVC5)/synthesized
Chalcone derivatives/synthesized
Chana chalcone derivatives/synthesized
Fluoro-substituted tris-chalcones derivatives (5a-5i)/
synthesized
Hydroxyl chalcones and bis-chalcones (1a-1m) and
(2a-2m)/synthesized
Prenylated chalcones (3, 4, 7) Flavanone-coupled
chalcones ( 9 , 12, 13)/natural from Boesenbergia
rotunda (L.) mansf
Chalcone-triazole derivatives/synthesized
Chalcone derivatives/Synthesized

Naphthylchalcones/synthesized

Xanthoangelol (XA) and 4-hydroxyderricin (4HD)/ natural from Angelica keiskei (miq.) koidz

Five derivatives from isoliquiritigenin (ISL)/natural from Glycyrrhiza glabra L

Chalcone derivatives: four DAs (morusalbins A-D)/ natural from Morus alba $\mathrm{L}$.

Chalcone 1/synthesized

Chalcones: BUT, ISL, DHC, HDMC, DCC, DCCP, $\mathrm{CMC}, \mathrm{CMCP} /$ synthesized

2- hydroxychalcone/synthesized

Lavandulylated chalcone/natural from Sophora flavescens aiton

Xanthohumol (XN)/natural from Humulus lupulus L

Chalcone derivatives/synthesized

Diarylsulfonylurea-chalcone hybrids/synthesized

Trans-chalcone (benzylideneacetophenone)

4-Hydroxyderricin (4HD) xanthoangelol (XAG)/natural 3T3-L1 adipocytes from Angelica keiskei (miq.) koidz

Chalcones AC1-AC11, BC1- BC6) 2', 4-dihydroxy chalcone-4-glucoside/synthesized and natural from Justicia adhatoda L

Chalcones $(6,7,20)$ /natural from Derris indica (lam.) a-Glucosidase inhibitory assay bennet

Sulfonylurea chalcones 1-3/synthesized

30-C-b-dglucopyranosyldihydro chalcone (22)/ synthesized

\section{Experimental Type of} model/method

STZ-induced diabetic rats study

a-Glucosidase inhibitory assay In vitro Glucose uptake in yeast cells In vitro

STZ-induced diabetic rats

In vivo

a-Glucosidase assay dipeptidyl In vitro peptidase-4 Adipocyte differentiation

a-Glucosidase inhibitory assay In vitro

a-Glucosidase assay Kinetics

of enzyme inhibition Glucose level

a-Glucosidase inhibitory assay In vitro

a-Glucosidase inhibitory assay In vitro

STZ-induced diabetic rats

In vivo

STZ-induced diabetic rats

In vivo

STZ-induced diabetic Mice

In vivo

STZ-induced diabetic Mice

a-Glucosidase inhibitory assay a-Glucosidase inhibitory assay

STZ-induced diabetic rats

HFD-induced diabetic rats

$\alpha$-Glucosidase $\beta$-amylase $\beta$-galactosidase $\alpha$-amylase inhibitory assays

a-Glucosidase inhibitory assay

a-Amylase a-glucosidase inhibitory assays

STZ-induced diabetic rats

STZ-induced diabetic Rats
Normoglycemic rabbits

STZ-induced diabetic rats

Measuring the glucose diffusion In vitro In vitro In vivo In vivo
In vivo
Anti-hyperglycemic: $\downarrow$ blood glucose level normalization of serum biochemical parameters 10-20 mg/kg, bw

$\downarrow$ a-glucosidase $\mathrm{IC}_{50}=15 \mathrm{mg} / \mathrm{ml}$

Chalcones MCV4, MCV5: $\uparrow$ glucose uptake

$\mathrm{IC}_{50}=5-15 \mathrm{mg} / \mathrm{ml}$

Anti-hyperglycemic: $\downarrow$ blood glucose level $10 \mathrm{mg} / \mathrm{kg}$ bw

Chana 1: $\downarrow$ a-glucosidase, $\downarrow$ DPP-4 $\uparrow$ adipocyte

differentiation $\mathrm{IC}_{5}=250 \mu \mathrm{M} / \mathrm{L}$

Chalcones 5a-5i: $\downarrow$ a-glycosidase $\mathrm{IC}_{50}=22.5 \mu \mathrm{M}$

$\downarrow$ a-glucosidase Chalcones 2c, 2g, 2j,2l, are noncompetitive inhibitors Chalcone2g: $\downarrow$ blood glucose level

$\downarrow$ a-glucosidase, $I_{50}=1.2-20 \mu \mathrm{g} / \mathrm{ml}$ 2017)

The most active chalcones: $4 \mathrm{~m}, \mathrm{IC}_{50}=67.78 \mu \mathrm{M}$ $4 \mathrm{p}, \mathrm{IC}_{50}=74.94 \mu \mathrm{M} 4 \mathrm{~s}, \mathrm{IC}_{50}=102.10 \mu \mathrm{M}$

(Chinthala et al., 2015) $\uparrow$ secretion of insulin No effects on glucose uptake (Damazio et al., into muscle No effects on blood glucose levels $50 \mathrm{mg} / \mathrm{kg}$ bw

$\uparrow$ glucose tolerance curve $\uparrow$ secretion of insulin $10 \mathrm{mg} / \mathrm{kg}$ bw

2009)

(Damazio et al., 2010)

(Enoki et al., 2010) secretion of insulin diet containing $0.15 \%$ chalcone $4 \mathrm{HD}$

In vivo Anti-hyperglycemic: $\downarrow$ blood glucose level $100 \mathrm{mg} / \mathrm{kg}$ bw

DAs $(1-4,6-8,11,12,14)$, DAs $(4,6-8)$ : $\downarrow$ a-glucosidase $\mathrm{IC}_{50}=2.25-5.90 \mu \mathrm{M}$ $\downarrow$ a-glucosidase, $\mathrm{IC}_{50}=840 \mu \mathrm{M}$, compared with acarbose $\mathrm{IC}_{50}=860.25 \pm 6.20 \mu \mathrm{M}$ $\downarrow$ glycogen content in liver $25 \mathrm{mg} / \mathrm{kg}$ bw

(Gaur et al., 2014)

(Ha et al., 2018)

(Imran et al., 2015)

(Jamal et al., 2009)

$\downarrow$ secretion of insulin $\uparrow$ glycosylated $\mathrm{hb}, \uparrow$ glucose blood level $25 \mathrm{mg} / \mathrm{kg}$ bw

In vitro $\quad \downarrow \beta$-galactosidase, $\mathrm{IC}_{50}=57 \mu \mathrm{M} \downarrow$ a-glucosidase, noncompetitive inhibition $\downarrow \beta$-amylase, mixed inhibition $\mathrm{IC}_{50}=57 \mu \mathrm{M}$

$\downarrow$ a-glucosidase; reversible, noncompetitive $\mathrm{IC}_{50}=8.8 \mu \mathrm{M}$

In vitro $\downarrow \alpha$-amylase, $\downarrow \alpha$-Glucosidase $\mid \mathrm{IC}_{50}=1250 \mu \mathrm{g} / \mathrm{ml}$

Anti-hyperglycemic: $\downarrow$ blood glucose level 10, 30, $50 \mathrm{mg} / \mathrm{kg}$ bw

In vivo Anti-hyperglycemic: $\downarrow$ blood glucose level $\uparrow$ moderate secretion of insulin 2, 8, 16, $32 \mathrm{mg} / \mathrm{kg}$ bw

In vitro Chalcones 4HD, XAG: $\uparrow$ glucose uptake GLUT4dependent through the LKB1/AMPK signaling pathway $\mathrm{IC}_{50}=20 \mu \mathrm{mol} / \mathrm{L}$

All chalcones: Good anti-hyperglycemic effect AC6: The highest activity $\mathrm{IC}_{50}=100 \mu \mathrm{g} / \mathrm{ml}$

(Jayanthi et al., 2012)

(Kim et al., 2006)

(Liu et al., 2014)

(Monisha et al. 2018)

(Naidu, 2018)

(Najafian et al.,

2010)

(Ohta et al., 2015)

$\downarrow$ a-glucosidase chalcone 6: $\mathrm{IC}_{50}=103.5 \mu \mathrm{M}$

(Purnima et al.

2012)

(Romagnoli et al., 2008)

All compounds: Hypoglycemic activity Compound- (Rao et al., 2014) 3: The highest activity (38.73\%) $5 \mathrm{mg} / \mathrm{kg}$ bw Chalcone 22: $\downarrow$ blood glucose (comparable to metformin), $25 \mathrm{mg} / \mathrm{kg}$ bw

(Rawat et al., 2011)

(Continued on following page) 
TABLE 1 | (Continued) Antidiabetic activities of chalcones: in vitro and in vivo preclinical pharmacological studies.

\begin{tabular}{|c|c|c|c|c|}
\hline Chalcones/source & $\begin{array}{l}\text { Experimental } \\
\text { model/method }\end{array}$ & $\begin{array}{l}\text { Type of } \\
\text { study }\end{array}$ & Results/mechanisms & Ref \\
\hline $\begin{array}{l}\text { 2', 4'- dihydroxy-4-methoxydihydrochalcone (DMC- } \\
\text { 2)/synthesized }\end{array}$ & $\begin{array}{l}\text { HFD obese C57BL/6J male } \\
\text { mice }\end{array}$ & In vivo & $\begin{array}{l}\downarrow \text { blood glucose (comparable to metformin) } \\
200-300 \mathrm{mg} / \mathrm{kg} \mathrm{bw}\end{array}$ & $\begin{array}{l}\text { (Ribnicky et al., } \\
\text { 2009) }\end{array}$ \\
\hline $\begin{array}{l}\text { Chalcones (1-4)/natural from Broussonetia } \\
\text { papyrifera (L.) L'Hér. Ex vent }\end{array}$ & a-Glucosidase inhibitory assay & In vitro & $\begin{array}{l}\text { Chalcones 1: } \downarrow \alpha \text {-glucosidase, } \mathrm{IC}_{50}=5.3 \mu \mathrm{M} \\
\text { Chalcones 2: } \downarrow \text {-glucosidase, } \mathrm{IC}_{50}=11.1 \mu \mathrm{M}\end{array}$ & (Ryu et al., 2010) \\
\hline Chalcones (5a-r), (4a-e), (3a-e)/synthesized & $\begin{array}{l}\text { HFD sucrose STZ-induced } \\
\text { diabetic rats }\end{array}$ & In vivo & $\begin{array}{l}\text { Chalcones 5a, g, m, o, p, r Anti-hyperglycemic: } \\
\text { \blood glucose level } 100 \text { mg/kg bw }\end{array}$ & $\begin{array}{l}\text { (Satyanarayana } \\
\text { et al., 2004) }\end{array}$ \\
\hline $\begin{array}{l}\text { Chalcone-6'-hydroxy-2',3,4-trimethoxy-4'-O- } \beta \text {-D- } \\
\text { glucopyranoside (1)/natural from Pouzolzia rugulosa } \\
\text { (wedd.) acharya and kravtsova }\end{array}$ & Alloxan-induced diabetic mice & In vivo & Hypoglycemic activity $100,200,500$ mg/kg bw & $\begin{array}{l}\text { (Semwal et al., } \\
\text { 2009) }\end{array}$ \\
\hline $\begin{array}{l}\text { 1-\{4-[(2E)-3-(substituted phenyl) prop-2- enoyl] } \\
\text { phenyl\}-3-(substituted phenyl") urea (2a-d), 3(a-c)/ } \\
\text { synthesized }\end{array}$ & STZ-induced diabetic Rats & In vivo & $\begin{array}{l}\text { Anti-hyperglycemic: } \downarrow \text { blood glucose level doses of } \\
\text { compounds } 2(a-d) \text { and (a-c) } 35 \mathrm{mg} / \mathrm{kg} \text { bw }\end{array}$ & $\begin{array}{l}\text { (Sengupta et al., } \\
\text { 2017) }\end{array}$ \\
\hline Chalcone derivatives $(1-20) /$ synthesized & $\begin{array}{l}\alpha \text {-Amylase, } \alpha \text {-glucosidase } \\
\beta \text {-amylase inhibitory assays }\end{array}$ & In vitro & $\begin{array}{l}\text { Chalcone 20: } \downarrow \text {-glucosidase } \mathrm{IC}_{50}=0.4 \mu \mathrm{M} \text {, non- } \\
\text { competitive inhibition }\end{array}$ & (Seo et al., 2005) \\
\hline Trihydroxychalcone derivatives/synthesized & $\begin{array}{l}\text { C2C12 myotubes cells HFD } \\
\text { diabetic C57BL/ } 6 \text { mice }\end{array}$ & $\begin{array}{l}\text { In vitro } \\
\text { In vivo }\end{array}$ & $\begin{array}{l}\text { Chalcone 13: } \uparrow \text { AMPK } \rightarrow \uparrow \text { AMP-activated } \\
\mathrm{C}_{50}=10 \mu \mathrm{mol} / \mathrm{L} \text { protein kinase; } \uparrow \text { glucose tolerance, } \\
\uparrow \text { muscle } \mathrm{FAO}, \downarrow \text { fat in skeletal muscles, liver } \\
30 \mathrm{mg} / \mathrm{kg} \mathrm{bw}\end{array}$ & (Shin et al., 2018) \\
\hline $\begin{array}{l}\text { Chalcone-based aryloxypropanolamines (5a-n)/ } \\
\text { synthesized }\end{array}$ & $\begin{array}{l}\text { HFD sucrose and STZ-induced } \\
\text { diabetic rats }\end{array}$ & In vivo & Anti-hyperglycemic: $\downarrow$ blood glucose level & (Shukla et al., 2007) \\
\hline $\begin{array}{l}\text { Chalcone-based aryloxy-propanolamines } 3,9(a, b) \text {, } \\
\text { 10/synthesized }\end{array}$ & $\begin{array}{l}\text { HFD sucrose and STZ-induced } \\
\text { diabetic rats }\end{array}$ & In vivo & $\begin{array}{l}\text { Chalcone 9a: } \uparrow \text { glucose tolerance in sucrose HFD } \\
\text { sucrose feeded rats Chalcones 3, 9a, 9b: } \uparrow \\
\text { postprandial hyperglycaemia in STZ-induced } \\
\text { diabetic rats } 100 \mathrm{mg} / \mathrm{kg} \mathrm{bw}\end{array}$ & (Shukla et al., 2017) \\
\hline $3^{\prime}, 5^{\prime}$-digeranylated chalcone (16)/synthesized & a-Glucosidase inhibitory assay & In vitro & $\begin{array}{l}\downarrow \alpha \text {-glucosidase, interaction chalcone } 16 \text { and } \\
\text { a-glucosidase's } \mathrm{IC}_{50}=0.90 \mu \mathrm{M}\end{array}$ & (Sun et al., 2015) \\
\hline Prenylchalconaringenins (1) and (2)/natural & $\begin{array}{l}\text { a-Amylase, a-glucosidase } \\
\text { inhibitory assays STZ-induced } \\
\text { diabetic mice }\end{array}$ & $\begin{array}{l}\text { In vitro } \\
\text { In vivo }\end{array}$ & $\begin{array}{l}3^{\prime} \text {-Geranylchalconaringenin (2) } \downarrow \text { a-amylase, } \\
\mathrm{IC}_{50}=20.46 \mu \mathrm{M} \downarrow \text { a-glucosidase, } I_{50}=1.08 \mu \mathrm{M} \\
\downarrow \text { postprandial blood glucose, } \downarrow T G, \downarrow \text { cholesterol } \\
60 \mathrm{mg} / \mathrm{kg} \text { bw }\end{array}$ & (Sun et al., 2017) \\
\hline Chalcones $(2 \mathrm{a}, 2 \mathrm{~b}, 2 \mathrm{c}) /$ synthesized & STZ-induced diabetic Rats & In vivo & $\begin{array}{l}\text { Chalcone 2a: } \downarrow \text { blood glucose level, anti- } \\
\text { hyperglycemic in diabetic rats Chalcone } 2 \mathrm{c} \text { : } \downarrow \text { blood } \\
\text { glucose level in normoglycemic rats } 100 \mathrm{mg} / \mathrm{kg} \mathrm{bw}\end{array}$ & $\begin{array}{l}\text { (Tajammal et al., } \\
\text { 2017) }\end{array}$ \\
\hline Chalcone units of conjugates/synthesized & a-Glucosidase inhibitory assay & In vitro & $\begin{array}{l}\text { All chalcones: } \downarrow \text { a-glucosidase Chalcone } 1 \mathrm{~b}: \uparrow \\
\text { inhibitory activity } \mathrm{IC}_{50}=3.2 \mu \mathrm{M}\end{array}$ & (Tang et al., 2014) \\
\hline $\begin{array}{l}2^{\prime}, 4^{\prime} \text {-dihydroxy- } 6^{\prime} \text {-methoxy- } 3^{\prime}, 5^{\prime} \text {-dimethylchalcone } \\
\text { (DMC)/natural from Cleistocalyx operculatus (roxb.) } \\
\text { merr. and L.M.Perry }\end{array}$ & a-Amylase inhibitory assay & In vitro & DMC: $\downarrow$ pancreatic $a$-amylase $\mathrm{IC}_{50}=69 \mu \mathrm{M}$ & $\begin{array}{l}\text { (Zhang and Lu, } \\
\text { 2012) }\end{array}$ \\
\hline Chalcone derivatives (13a-h), (19a-h)/synthesized & STZ-induced diabetic Mice & In vivo & $\begin{array}{l}\text { Chalcones 13e, 13g, 19f; } \downarrow \text { TG, } \downarrow \text { TC, } \downarrow \text { Glu } \\
\text { Chalcones 13e, 19f: } \uparrow \text { AMPK, } \uparrow P P A R a 50 \mathrm{mg} / \mathrm{kg} \text { bw }\end{array}$ & (Zhu et al., 2018) \\
\hline
\end{tabular}

Abbreviations and symbols: $\uparrow$, increased; $\downarrow$, decreased; STZ, streptozotocin; MD, molecular dynamic simulations; HFD, high fructose diet; GLUT-4, glucose transporter type 4; LKB1, liver kinase B1; AMPK, AMP-activated protein kinase; PPAR $\alpha$, peroxisome proliferator-activated receptors; BW, body weight.

(Orlikova et al., 2012). Isoliquiritigenin also reduced palmitic acid-induced macrophage activation, leading to additional antiinflammatory activity (Watanabe et al., 2016). In human primary endothelial cells Isoliquiritigenin prevented the translocation and stimulation of NF- $\kappa \mathrm{B}$ by hindering the phosphorylation and subsequent decomposition of IkBa (Kumar et al., 2007).

\section{Antimicrobial and Antifungal Activity}

From the leaves and stems of Crotalaria madurensis Wight \& Arn., crotmadine (1) was isolated that exhibited antifungal activity (Bhakuni and Chaturvedi, 1984). Five prenylated flavonoids, including one new natural product (2-6), were isolated from an ethanol extract of the leaves of Maclura tinctoria (L.) D. Don ex Steud. All the isolated compounds were evaluated against Candida albicans and Cryptococcus neoformans. Compound 3 (isobavachalcone) was found to be the most active against both the yeasts (ElSohly et al., 2001). The crude methanolic extract of Zuccagnia angulata Hook. and Arn. by assay guided fractionation led to the isolation of two chalcones (7-8) as the compounds responsible for the antifungal activity (Svetaz et al., 2004). The antifungal activity of the chalcones (9-13), extracted from the methanol extract of the leaves of Artocarpus nobilis Thwaites, showed potent fungicidal activity (Jayasinghe et al., 2004). A new dimeric chalcone (14) isolated from the fresh whole uncrushed fruits of Mallotus philippinensis var. pallidus Airy Shaw was evaluated for antifungal susceptibility with good results (Kulkarni et al., 2014). The extracted compounds from Zuccagnia punctata Cav. were found to be efficacious as inhibitors of Candida species (Gabriela et al., 2014). In a recent study, the antifungal activity of 40 synthetized chalcones and analogs (20-59) was analyzed. Chalcones with different substituents showed to be active against different tested 
TABLE 2 | Anti-inflammatory activities of chalcones.

\section{Chalcones/source}

5'-Chloro-2'-hydroxy-4'6'-dimethyl-3, 4, 5 -Trimethoxy-chalcone/synthesized

3-(5-bromo-1H-indol-3-yl)-1-(4-cyanophenyl) prop-2-en-1-one/synthesized

(5-Methoxy-1H-indol-3-yl)-1-(4-(methylsulfonyl) phenyl) prop-2-en-1-one/ synthesized

Hydroxy-3,4,6-trimethoxychalcone/natural from Toussaintia orientalis verdc

Licochalcone A/natural from Glycyrrhiza inflata batalin

(E)-3-(4-((ethylamino)methyl)-phenyl) -1-(5-methylfuran-2-yl)prop-2-en-1-one/ synthesized

Ferrocenyl-3-(4-methylsulfonylphenyl) propen-1-one/synthesized

(E)-4-methyl-N-((4-(3-(3,4,5 trimethoxyphenyl) acryloyl)phenyl)-carbamoyl) benzenesulfonamide (Me-UCH5)/synthesized

(E)-1-(2,6-dimethoxyphenyl)-3-(4-(dimethylamino)phenyl)prop-2-en-1-one/ synthesized

(E)-1-(2,5-dimethoxyphenyl)-3-(4-(dimethylamino)phenyl)prop-2-en-1-one/ synthesized

3,4,5-Trimethoxy-4'-fluorochalcone/synthesized

1-[6-(3,7-dimethyl-octa-2,6-dienyl)-5,7-dihydroxy-2,2-dimethyl-2H-chromen-8yl]-3-(4-hydroxy-phenyl)- propanone/natural Mallotus philippinensis

3-(3,4-dihydroxy-phenyl)-1-[6-(3,7-dime-thyl-octa-2,6-dienyl)-5,7-dihydroxy-2,2- $\quad \downarrow$ PGE2 dimethyl-2H-chromen- 8-yl]-propenone/natural Mallotus philippinensis

1-[5,7-dihydroxy-2-methyl-6-(3-methyl-but-2-enyl)-2-(4-methyl-pent-3-enyl)-2H- $\quad \downarrow P G E 2$ chromen-8-yl]-3-(3,4- dihydroxy-phenyl)-propenone/natural Mallotus philippinensis

Broussochalcone A/natural from Broussonetia papyrifera (L.) L'Hér. Ex vent

Isobavachalcone/natural from Cullen corylifolium (L.) medik

Bavachromene/natural from Cullen corylifolium (L.) medik

Kanzonol B/natural from Cullen corylifolium (L.) medik

(3-(2-Hydroxyphenyl)-1-(thiophene-3-yl)prop-2-en-1-one) (TI-I-174)/synthesized

2-(3-(3,4-dimethoxyphenyl)propyl)-5-methoxyphenol/synthesized

(E)-1-(4-hydroxy-3-methoxyphenyl)-3-(3,4,5-trimethoxyphenyl)prop-2-en-1-one/ synthesized

(E)-1-(3-methoxyphenyl)-3-(3,4,5-trimethoxyphenyl)prop-2-en-1-one/synthesized

2'-methoxy-3,4-dichlorochalcone/synthesized

$2^{\prime}$-hydroxy-6'-methoxychalcone/synthesized

$2^{\prime}$-hydroxy-3-bromo-6'-methoxychalcone/synthesized

2'-hydroxy-4',6'-dimethoxychalcone/synthesized

2', 5', -dihydroxy-4-chloro-dihydrochalcone/synthesized

4-hydroxylonchocarpin/natural from Psoralea corylifolia L

\begin{tabular}{lll}
\multicolumn{1}{c}{ Mechanism } & \multicolumn{1}{c}{ Results } & \multicolumn{1}{c}{ Ref } \\
$\downarrow$ COX-1 $\downarrow$ COX-2 & $\mathrm{IC}_{50}=87.6 \mu \mathrm{M} \mathrm{IC} \mathrm{C}_{50}=88.0 \mu \mathrm{M}$ & (Bano et al., 2013) \\
$\downarrow$ TNF- $\alpha$ & $\mathrm{IC}_{50}=5-10 \mu \mathrm{M}$ & \\
$\downarrow$ COX-1 $\downarrow$ COX-2 & $\mathrm{IC}_{50}=23.2 \pm 0.5 \mu \mathrm{g} / \mathrm{ml}$ & (Özdemir et al., 2015) \\
& $\mathrm{IC}_{50}=27.1 \pm 2.5 \mu \mathrm{g} / \mathrm{ml}$ & \\
$\downarrow$ COX-1 & $\mathrm{IC}_{50}=24.5 \mu \mathrm{g} / \mathrm{ml}$ no effect on & (Özdemir et al., 2015) \\
& $\mathrm{COX}-2$ &
\end{tabular}

$\downarrow$ COX -1

$\mathrm{IC}_{50}=9565 \mu \mathrm{g} / \mathrm{ml}$ no effect on

(Nyandoro et al., 2012) COX-2

$\downarrow \mathrm{COX}-1 \downarrow \mathrm{COX}-2$

$\mathrm{IC}_{50}=0.94 \mu \mathrm{g} / \mathrm{ml} \mathrm{C}_{50}=1.93 \mu \mathrm{g} / \mathrm{ml}$

$\mathrm{IC}_{50}=25.85 \mu \mathrm{g} / \mathrm{ml} \mathrm{IC}_{50}=$

$10.08 \mu \mathrm{g} / \mathrm{ml}$

$\downarrow$ COX-2

$\mathrm{IC}_{50}=0.05 \mu \mathrm{g} / \mathrm{ml}$ no effect on COX-1

$\downarrow$ COX-2

$\downarrow$ PGE2

$\mathrm{IC}_{50}=0.06 \mu \mathrm{g} / \mathrm{ml}$ no effect on COX-1

$\downarrow$ PGE2

$\mathrm{IC}_{50}=0.6 \mu \mathrm{M}$

$\mathrm{IC}_{50}=0.7 \mu \mathrm{M}$

$\mathrm{IC}_{50}=0.033 \mu \mathrm{M}$

$\mathrm{IC}_{50}=7.6 \mu \mathrm{M}$

$\mathrm{IC}_{50}=9.5 \mu \mathrm{M}$

$\mathrm{IC}_{50}=38.6 \mu \mathrm{M}$

(Okuda-Tanino et al., 2017)

(Jantan et al., 2014)

(Farzaneh et al., 2018)

(Araico et al., 2006)

(Rojas et al., 2002)

(Rojas et al., 2002)

(Rojas et al., 2003b)

(Daikonya et al., 2004)

(Daikonya et al., 2004)

(Daikonya et al., 2004)

$\downarrow$ PGE2

$\downarrow$ PGE2

$\downarrow$ PGE2

$\downarrow$ PGE2

$\downarrow$ PGE2

$\downarrow$ PGE2

$\downarrow$ PGE2

$\downarrow$ PGE2

$\downarrow$ PGE2

$\downarrow$ PGE2

$\downarrow$ PGE2

$\downarrow$ PGE2

$\downarrow$ PGE2

$\downarrow$ PGE2

$$
\begin{aligned}
I C_{50} & =11.3 \mu \mathrm{M} \\
I C_{50} & =1.6 \pm 0.11 \mu \mathrm{M} \\
I C_{50} & =2.4 \pm 0.18 \mu \mathrm{M} \\
I C_{50} & =2.2 \pm 0.21 \mu \mathrm{M} \\
I C_{50} & =5.75 \mu \mathrm{M} \\
I C_{50} & =6.5 \mu \mathrm{M} \\
I C_{50} & =4.19 \mu \mathrm{M} \\
I C_{50} & =2.88 \mu \mathrm{M} \\
I C_{50} & =7.1 \mu \mathrm{M} \\
I C_{50} & =9.6 \mu \mathrm{M} \\
I C_{50} & =7.8 \mu \mathrm{M} \\
I C_{50} & =9.6 \mu \mathrm{M} \\
I C_{50} & =4.0 \pm 1.5 \mu \mathrm{M} \\
I C_{50} & =10.2 \mu \mathrm{M}
\end{aligned}
$$

fungi probably by inhibiting the biosynthesis of one or both polymers of the fungal cell wall (Lopez et al., 2001). A large series of chalcones were synthesized and studied for antifungal activity against Candida albicans; the chalcones (60-64) exhibited promising anti-candidal activities (Batovska et al., 2007).

As part of ongoing studies in developing new antimicrobials, ten new thiazole-based chalcones (77-86) were synthesized and tested for their in vitro antifungal properties. These possessed modest activity against all the fungal species tested and were being less active than ketoconazole and bifonazole (Liaras et al., 2011). The chromonyl chalcones (87-88) were used as intermediates for the synthesis of new bioactive pyrazoline derivatives (89-94) under green condition. The antifungal and antimicrobial activity was tested by disk diffusion assay. The maximum inhibition was observed by chalcones 84 and 89 against $S$. aureus (Siddiqui et al., 2012). Using the agar cupplate method, the antimicrobial activities of the synthesized compounds (95-106) were screened in vitro. The results exhibited promising antifungal activity and antibacterial activity (Prasath et al., 2013). Compound 107 was evaluated for its antibacterial properties and showed maximum zone of inhibition against $S$. aureus and $P$. aeruginosa (Bhale et al., 2013). A series of a-triazolyl chalcones were synthesized (108-121), and the synthesized compounds showed potent antibacterial activity 
TABLE 3 | Antileishmanial activity of chalcones.

\begin{tabular}{|c|c|c|c|c|}
\hline Chalcones/source & Type of study & Teste & d effects Parasite & Ref \\
\hline Licochalcone/natural & In vitro & $\begin{array}{l}L . \text { donovani } \\
\text { promastigotes } \\
\text { amastigote form of } L \text {. } \\
\text { major }\end{array}$ & $\mathrm{IC50}=2.4 \mu \mathrm{g} / \mathrm{ml}$ & (Chen et al., 1993) \\
\hline $\begin{array}{l}\left.2^{\prime}, 6^{\prime} \text {-dihydroxy-4'-methoxychalcone (DMC, } 2\right) / \\
\text { natural }\end{array}$ & In vitro & $\begin{array}{l}\text { L. amazonensis } \\
\text { promastigotes }\end{array}$ & $\begin{array}{l}\text { Damages of cell ultrastructure } \\
\text { IC50 = 50 } \mu \mathrm{g} / \mathrm{ml}: \text { Damage to } \\
\text { amastigote mitochondria IC } 50=40 \mu \mathrm{g} / \mathrm{ml} \text { : } \\
\text { Damage to promastigote mitochondria }\end{array}$ & $\begin{array}{l}\text { (Torres-Santos et al., } \\
\text { 1999) }\end{array}$ \\
\hline $\begin{array}{l}\text { Dihydrochalcones, } 2^{\prime}, 6^{\prime} \text {-dihydroxy-4'- } \\
\text { methoxydihydrochalcone } 4 / \text { natural }\end{array}$ & In vitro & $\begin{array}{l}\text { L. infantum } \\
\text { promastigotes }\end{array}$ & $\mathrm{IC50}=15.30 \mu \mathrm{g} / \mathrm{ml}$ & (Hermoso et al., 2003) \\
\hline $\begin{array}{l}2^{\prime}, 6 \text { ', } 4 \text {-trihydroxy-4'-methoxydihydro chalcone (5)/ } \\
\text { natural }\end{array}$ & In vitro & $\begin{array}{l}\text { L. tropica promastigotes } \\
\text { L. infantum } \\
\text { promastigotes }\end{array}$ & $\mathrm{IC} 50=3.82 \mu \mathrm{g} / \mathrm{ml} \mathrm{IC} 50=6.35 \mu \mathrm{g} / \mathrm{ml}$ & (Hermoso et al., 2003) \\
\hline $\begin{array}{l}\text { Chalcones from Psorothamnus arborescens (A.Gray) } \\
\text { barneby/natural }\end{array}$ & In vitro & L. donovani amastigotes & $\mathrm{IC50}=5.0 \mu \mathrm{g} / \mathrm{ml}$ & $\begin{array}{l}\text { (Salem and Werbovetz, } \\
\text { 2005) }\end{array}$ \\
\hline Isoliquiritigenin/natural & In vitro & L. donovani amastigotes & $\mathrm{IC50}=5.30 \mu \mathrm{g} / \mathrm{ml}$ & $\begin{array}{l}\text { (Salem and Werbovetz, } \\
\text { 2006) }\end{array}$ \\
\hline $\begin{array}{l}\text { Chalcone from Lonchocarpus guatemalensis benth } \\
\text { natural }\end{array}$ & In vitro & $\begin{array}{l}\text { L. braziliensis } \\
\text { promastigotes }\end{array}$ & $\mathrm{IC50}=10 \mu \mathrm{g} / \mathrm{ml}$ & (Borges-Argaez et al., 2007) \\
\hline Chalcone-triclosan hybrids/semisynthetic & In vitro & L. panamensis & $\mathrm{IC} 50=9.4 \pm 1.3 \mu \mathrm{M}$ & (Otero et al., 2014) \\
\hline $2^{\prime}, 4^{\prime}$-dihydroxychalcone 35/synthesized & In vitro & $\begin{array}{l}\text { L. amazonensis } \\
\text { promastigotes }\end{array}$ & $\mathrm{IC50}=0.4 \mu \mathrm{M}$ & (Passalacqua et al., 2015) \\
\hline Methoxychalcones/synthesized & In vitro & $\begin{array}{l}\text { L. braziliensis } \\
\text { promastigote }\end{array}$ & $\mathrm{IC} 50<10 \mu \mathrm{M}$ & (Bello et al., 2011) \\
\hline $\begin{array}{l}\text { (1E,4E)-1,5-bis(3,4,5-trimethoxy-phenyl)-penta-1,4- } \\
\text { dien-3- one/synthesized }\end{array}$ & In vitro & L. Niannia) braziliensis & $\mathrm{IC} 50=1.38 \pm 1.08 \mu \mathrm{M}$ & (de Mello et al., 2014) \\
\hline $\begin{array}{l}\text { (1E,4E)-1,5-bis(phenyl)-penta-1,4-dien-3-one/ } \\
\text { synthesized }\end{array}$ & In vitro & L. Niannia) braziliensis & $\mathrm{IC50}=5.88 \pm 1.35 \mu \mathrm{M}$ & (de Mello et al., 2014) \\
\hline $\begin{array}{l}\text { (2E)-1-phenyl-3-(3,4,5-trimethoxy-phenyl)-prop-2- } \\
\text { en-1- one/synthesized }\end{array}$ & In vitro & L. Niannia) braziliensis & $\mathrm{IC} 50=6.36 \pm 2.04 \mu \mathrm{M}$ & (de Mello et al., 2014) \\
\hline $\begin{array}{l}\text { (2E)-1-(4-methoxy-phenyl)-3-(3,4,5-trimethoxy- } \\
\text { phenyl)- prop-2-en-1-one/synthesized }\end{array}$ & In vitro & L. Niannia) braziliensis & $\mathrm{IC} 50=5.69 \pm 0.20 \mu \mathrm{M}$ & (de Mello et al., 2014) \\
\hline Chalcone 22 Chromenochalcones/synthesized & In vivo & $\begin{array}{l}\text { L. donovani/hamster } \\
\text { model }\end{array}$ & $\begin{array}{l}50 \mathrm{mg} / \mathrm{kg} / \text { day } \rightarrow \downarrow \text { parasites } 48.53 \pm \\
10.43 \% \text { on day } 7 \text { post treatment }\end{array}$ & (Gupta et al., 2014) \\
\hline Chalcone 37 Chromenochalcones/synthesized & In vivo & $\begin{array}{l}\text { L. donovani/hamster } \\
\text { model }\end{array}$ & $\begin{array}{l}50 \mathrm{mg} / \mathrm{kg}, \text { for } 10 \text { days } \rightarrow \downarrow \text { parasites } \\
(83.32 \pm 12.37 \%)\end{array}$ & (Gupta et al., 2014) \\
\hline Chalcone-triclosan hybrids/semisynthetic & In vitro & L. panamensis & $\mathrm{IC50}=9.4 \pm 1.4 \mu \mathrm{g} / \mathrm{ml}$ & (Otero et al., 2014) \\
\hline
\end{tabular}

and antifungal activity (Yin et al., 2014). A new series of pyrazine analogs of chalcones have been tested against fungal strains. The results showed that the compounds were inactive or only weekly active against most strains (Kucerova-Chlupacova et al., 2015). In another study, a series (132-179) of isatin-ferrocenyl chalcone and isatin-ferrocene conjugates were synthesized and were evaluated for their inhibitory activities against $T$. vaginalis. The compounds exhibited 100\% growth inhibition (Singh et al., 2018). In another study, three chalcones, diuvaretin, uvaretin, and isouvaretin, were investigated on their antibacterial activity, and the culture inhibition was only observed for Gram-positive germs (Koudokpon et al., 2018). A series of ten chalcones and five new dihydrochromane-chalcone hybrids (189-203) were synthesized, and their antifungal activity was evaluated in vitro, and only two compounds had similar antifungal activity to that of the positive control (Mellado et al., 2019). A series of five fluorinated chalcones (204-208) were evaluated for their antibacterial activity against Gram-positive and Gram-negative pathogenic bacterial strains using the agar diffusion method. The results showed that the compounds exhibited broad-spectrum activity against these pathogens (Amole et al., 2019).

\section{Antiparasitic Activity Antileishmanial Activity}

The in vitro antileishmanial activity of chalcones was evaluated by several studies (Torres-Santos et al., 1999; Salem and Werbovetz, 2005; Salem and Werbovetz, 2006; Lima et al., 2016).

Licochalcone inhibited the growth of both Leishmania major and Leishmania donovani promastigotes and amastigotes and reduced the infection rate of human peripheral blood monocytederived macrophages (Chen et al., 1993). Adunchalcone displayed $50 \%$ effective concentrations against the promastigote forms of Leishmania (L.) amazonensis, $L$ (V.) braziliensis, $L$ (V.) shawi, and $L$ (L.) chagasi, respectively (Dal Picolo et al., 2014). In another study, chalcones obtained Psorothamnus polydenius (S.Watson) Rydb., and exhibited leishmanicidal properties (Salem and Werbovetz, 2005). The chalcone 2,6'-Dihydroxy-4'-methoxychalcone (DMC) showed significant activity against promastigotes and intracellular amastigotes of Leishmania amazonensis (Torres-Santos et al., 1999). Many other chalcone-derived plants displayed varying degrees of leishmanicidal activity such as isoliquiritigenin (Salem and Werbovetz, 2006), chalcone from Lonchocarpus xuul Lundell 
TABLE 4 | Antimalarial activity of chalcones.

\begin{tabular}{|c|c|c|c|c|c|c|}
\hline Chalcones & Source & Method & $\begin{array}{l}\text { Type } \\
\text { of } \\
\text { study }\end{array}$ & Parasite & Effects & Ref \\
\hline Bartericin A1 & Natural & $\begin{array}{l}\text { Culture W2 strain of } P \text {. } \\
\text { falciparum }\end{array}$ & In vitro & P. falciparum & $\mathrm{IC}_{50}=2.15 \pm 0.02 \mu \mathrm{M}$ & $\begin{array}{l}\text { (Ngameni et al., } \\
\text { 2007) }\end{array}$ \\
\hline Bartericin B2 & Natural & $\begin{array}{l}\text { Culture W2 strain of } P \text {. } \\
\text { falciparum }\end{array}$ & In vitro & P. falciparum & $\mathrm{IC}_{50}=19.27 \pm 0.06 \mu \mathrm{M}$ & $\begin{array}{l}\text { (Ngameni et al., } \\
\text { 2007) }\end{array}$ \\
\hline Stipulin 3, 4 & Natural & $\begin{array}{l}\text { Culture W2 strain of } P \text {. } \\
\text { falciparum }\end{array}$ & In vitro & P. falciparum & $\mathrm{IC}_{50}=5.13 \pm 0.04 \mu \mathrm{M}$ & $\begin{array}{l}\text { (Ngameni et al., } \\
\text { 2007) }\end{array}$ \\
\hline Hydroxylonchocarpin 4 & Natural & $\begin{array}{l}\text { Culture against the W2 strain } \\
\text { of } P . \text { falciparum }\end{array}$ & In vitro & P. falciparum & $\mathrm{IC}_{50}=3.36 \pm 0.07 \mu \mathrm{M}$ & $\begin{array}{l}\text { (Ngameni et al., } \\
\text { 2007) }\end{array}$ \\
\hline Isobavachalcone 5 & Natural & $\begin{array}{l}\text { Culture against the W2 strain } \\
\text { of } P . \text { falciparum }\end{array}$ & In vitro & P. falciparum & $\mathrm{IC}_{50}=19.00 \pm 0.02 \mu \mathrm{M}$ & $\begin{array}{l}\text { (Ngameni et al., } \\
\text { 2007) }\end{array}$ \\
\hline Kanzonol B & Natural & $\begin{array}{l}\text { Culture against the W2 strain } \\
\text { of } P . \text { falciparum }\end{array}$ & In vitro & P. falciparum & $\mathrm{IC}_{50}=9.63 \pm 0.04 \mu \mathrm{M}$ & $\begin{array}{l}\text { (Ngameni et al., } \\
\text { 2007) }\end{array}$ \\
\hline Cajachalcone & Natural & $\begin{array}{l}\text { The bioassay-guided } \\
\text { fractionation of methanol } \\
\text { extract of C. cajan leaves }\end{array}$ & In vitro & P. falciparum & $\mathrm{IC}_{50}=2.0 \mu \mathrm{g} / \mathrm{mL}$ & $\begin{array}{l}\text { (Ajaiyeoba et al., } \\
\text { 2013) }\end{array}$ \\
\hline Xanthohumol and seven derivatives & $\begin{array}{l}\text { Semi - } \\
\text { Synthetic }\end{array}$ & - & In vitro & P. falciparum & $\begin{array}{l}I_{50}=8.4 \pm 0.3 \mu \mathrm{M}(\mathrm{poW}) \\
\mathrm{IC}_{50}=24.0 \pm 0.7 \mu \mathrm{M}(\mathrm{Dd} 2)\end{array}$ & $\begin{array}{l}\text { (Frölich et al., } \\
\text { 2009) }\end{array}$ \\
\hline Sulfonamide chalcone derivatives & Synthetic & $\begin{array}{l}\text { Culture of } P \text {. falciparum } \\
\text { parasites }\end{array}$ & In vitro & P. falciparum & $\mathrm{IC}_{50}>10 \mu \mathrm{M}$ & $\begin{array}{l}\text { (Domínguez } \\
\text { et al., 2005) }\end{array}$ \\
\hline Sulfonamide chalcone derivatives & Synthetic & b-hematin formation & In vitro & P. falciparum & $\mathrm{IC}_{50}=0.48 \mu \mathrm{M}$ & $\begin{array}{l}\text { (Domínguez } \\
\text { et al., 2005) }\end{array}$ \\
\hline Quinolinyl chalcones derivatives & Synthetic & $\begin{array}{l}\text { Culture of } P \text {. falciparum } \\
\text { parasites }\end{array}$ & In vitro & P. falciparum & $\mathrm{IC}_{50}=19.0 \mu \mathrm{M}$ & $\begin{array}{l}\text { (Domínguez } \\
\text { et al., 2001) }\end{array}$ \\
\hline Hlorovinyl sulfone-like chalcone derivatives & Synthetic & $\begin{array}{l}\text { Claisen-Schmidt } \\
\text { condensation }\end{array}$ & In vitro & P. falciparum & $\mathrm{IC}_{50}=0.025-10 \mathrm{mM}$ & $\begin{array}{l}\text { (Dominguez } \\
\text { et al., 2009) }\end{array}$ \\
\hline Phenylurenyl chalcone & Synthetic & - & In vitro & P. falciparum & $\mathrm{IC}_{50}=1.76 \mu \mathrm{M}$ & $\begin{array}{l}\text { (Domínguez } \\
\text { et al., 2005) }\end{array}$ \\
\hline $\begin{array}{l}\text {-(2,5-dichlorophenyl)-3-(4-quinolinyl)-2- } \\
\text { propen-1-one }\end{array}$ & Synthetic & - & In vitro & P. falciparum & $\mathrm{IC}_{50}=200 \mathrm{nM}$ & (Li et al., 1995) \\
\hline Chloroquinoline & Synthetic & $\begin{array}{l}\text { Claisen-Schmidt } \\
\text { condensation }\end{array}$ & In vitro & P. falciparum & $\mathrm{IC}_{50}=31.54 \mathrm{mM}$ & $\begin{array}{l}\text { (Hayat et al., } \\
\text { 2011) }\end{array}$ \\
\hline $\begin{array}{l}\text { 1-(4-Benzimidazol-1-yl-phenyl)-3-(2, 4- } \\
\text { dimethoxy-phenyl)-propen-1-one }\end{array}$ & Synthetic & $\begin{array}{l}\text { Claisen-Schmidt } \\
\text { condensation }\end{array}$ & In vitro & P. falciparum & $\mathrm{IC}_{50}=1.1 \mu \mathrm{g} / \mathrm{ml}$ & $\begin{array}{l}\text { (Yadav et al., } \\
\text { 2012) }\end{array}$ \\
\hline Licochalcone & Synthetic & - & In vitro & P. falciparum & $\mathrm{IC}_{50}=1.43 \mu \mathrm{g} / \mathrm{ml}$ & $\begin{array}{l}\text { (Yadav et al., } \\
\text { 2012) }\end{array}$ \\
\hline Acridinyl chalcone derivatives (1a-k) & Synthetic & $\begin{array}{l}\text { Noncatalyzed nucleophilic } \\
\text { aromatic }\end{array}$ & In vitro & p falciparum & $\mathrm{IC}_{50}=2 \mathrm{mg} / \mathrm{ml}$ & $\begin{array}{l}\text { (Tomar et al., } \\
\text { 2010) }\end{array}$ \\
\hline $\begin{array}{l}\text { Chalcone-AZT hybrid series } 7 \text { and } \\
\text { 9Acetylenic chalcones (1a-c, 2a-e) } \\
\text { Chalcone-chloroquinoline hybrid } \\
\text { compounds ( } 8 \text { and } 10)\end{array}$ & Synthetic & - & In vitro & p falciparum & $\begin{array}{l}\text { Compound } 8 \mathrm{~b} \text { was the most active, } \\
\text { submicromolar } \mathrm{IC}_{50} \text { values against the } \\
\mathrm{D} 10, \mathrm{Dd} 2 \text { and } \mathrm{W} 2 \text { strains of } P \text {. } \\
\text { falciparum. }\end{array}$ & $\begin{array}{l}\text { (Guantai et al., } \\
\text { 2010) }\end{array}$ \\
\hline Alkoxylated Chalcones & Synthetic & - & In vitro & P. falciparum & $\mathrm{IC}_{50}=6.5 \mathrm{mM}$ & $\begin{array}{l}\text { (Nowakowska, } \\
\text { 2007) }\end{array}$ \\
\hline 4-Chloro-20,40-dihydroxychalcone & Synthetic & - & In vitro & P. falciparum & $\mathrm{IC}_{50}=12.3 \mathrm{mM}$ & $\begin{array}{l}\text { (Nowakowska, } \\
\text { 2007) }\end{array}$ \\
\hline Hydroxylated chalcones & Synthetic & - & In vitro & P. falciparum & $\mathrm{IC}_{50}=20 \mathrm{mM}$ & $\begin{array}{l}\text { (Nowakowska, } \\
\text { 2007) }\end{array}$ \\
\hline Phenylurenyl chalcone derivatives & Synthetic & - & In vitro & P. falciparum & $\mathrm{IC}_{50}=1.75-10 \mathrm{mM}$ & $\begin{array}{l}\text { (Nowakowska, } \\
\text { 2007) }\end{array}$ \\
\hline Xanthohumol & Synthetic & - & In vitro & P. falciparum & $\mathrm{IC}_{50}=8.2 \mathrm{mM} \mathrm{IC}_{50}=24 \mathrm{mM}$ & $\begin{array}{l}\text { (Nowakowska, } \\
\text { 2007) }\end{array}$ \\
\hline
\end{tabular}

(Borges-Argaez et al., 2007), chalcones from Calea uniflora Less (family Compositae) (Lima et al., 2016), and sulfonamide 4methoxychalcone derivatives (Andrighetti-Fröhner et al., 2009). A series of oxygenated chalcones demonstrated remarkable antileishmanial activity (Liu et al., 2003). The compound derived from triclosan was evaluated for antileishmanial activity against $L(\mathrm{~V})$ panamensis amastigotes, and the compound was found to be active against Leishmania parasites
(Otero et al., 2014). The compounds of methoxychalcones and another synthetic chalcone, 2',4'-dihydroxychalcone displayed potent in vitro antileishmanial activity (Bello et al., 2011; Passalacqua et al., 2015). Also, chalcones (1-4) displayed potent leishmanicidal activity via reducing the infection index of macrophages significantly (De Mello et al., 2014).

In vivo, licochalcone $\mathrm{A}$ has completely prevented lesion development in L. major-infected mice (Chen et al., 1994; 
TABLE 5 | Cytotoxic and antiproliferative activity of chalcones.

\begin{tabular}{|c|c|c|c|c|}
\hline Chalcones & Source & Type of study & Effects & Ref. \\
\hline Chalcones with piperazine moiety. & Synthetic & $\begin{array}{l}\text { In vitro (different cancer } \\
\text { cells) }\end{array}$ & Anticarcinogenic properties & $\begin{array}{l}\text { (Filosa et al., 2007) } \\
\text { (Rahaman et al., 2010) }\end{array}$ \\
\hline Imidazoquinonyl chalcones and pyrazolines. & Synthetic & In vitro (HeLa cells) & Anticarcinogenic properties & (Viveka et al., 2014) \\
\hline$\beta$-carboline based chalcones. & Synthetic & In vitro (MCF-7 cells) & DNA fragmentation and apoptosis & (Chauhan et al., 2014) \\
\hline Heteroaromatic chalcones. & Synthetic & In vitro (T47D cells) & Topoisomerases inhibitory and cytotoxic activity & (Jeon et al., 2016) \\
\hline $\begin{array}{l}\text { Chalcone derived compounds replaced } \\
\text { acetophenone and replaced aldehyde. }\end{array}$ & Synthetic & In vitro (MCF-7 cells) & $\begin{array}{l}\text { Apoptosis induction in MCF-7 cells with the involvement } \\
\text { of caspase-7, caspase-8, and caspase- } 9\end{array}$ & (Syam et al., 2012) \\
\hline Thiophene analogues of chalcones. & Synthetic & In vitro (K562 cells) & Inhibition of Tubulin polymerization & (Romagnoli, 2008) \\
\hline $\begin{array}{l}\text { Chalcone derived compounds Hsp90 } \\
\text { inhibitors }\end{array}$ & Synthetic & $\begin{array}{l}\text { In vitro (H1975 and } \\
\text { MDA-MB-231 cells) }\end{array}$ & HSP90 inhibitory effect & $\begin{array}{l}\text { (Jeong et al., 2014; Oh and } \\
\text { Seo, 2017) }\end{array}$ \\
\hline
\end{tabular}

Tajuddeen et al., 2018). Chromenochalcones also showed antileishmanial potential in hamster (Gupta et al., 2014). Oral administration of chalcone 3-nitro-2-hydroxi-4,6dimetoxychalcone (CH8) in the groups of animals infected with either Leishmania infantum or Leishmania amazonensis showed good effect (Sousa-Batista et al., 2018).

Table 3 summarizes the antileishmanial effects of chalcones using in vitro and in vivo approaches.

\section{Antimalarial Activity}

Naturally occurring chalcones have demonstrated promising potencies after being tested in vitro against Plasmodium falciparum. The compounds bartericin A, stipulin 3, 4, and hydroxylonchocarpin demonstrated particular antimalarial potential with relatively low doses (Ngameni et al., 2007). Other chalcones with antimalarial activity proved in vitro: cajachalcone (Ajaiyeoba et al., 2013), xanthohumol (Frölich et al., 2009), sulfonamide chalcone derivatives (Domínguez et al., 2005), sythesized novel chlorovinyl sulfone-like chalcone derivatives (Dominguez et al., 2009), quinolinyl chalcones synthesized (Domínguez et al., 2001), various 1,3-diaryl-2-propenones (chalcone derivatives) (Geyer et al., 2009), and chalcone chloroquinolines such as chloroquine and quinine (Hayat et al, 2011). Among the 27 novel chalcone derivatives synthesized, only one compound was found to be the most antimalarial active (Yadav et al., 2012).

Chalcone derivatives administrated intraperitoneally to the Plasmodium yoelii-infected mice model showed significant inhibition of these strains (Tomar et al., 2010).

Table 4 summarizes the principal studies carried out on the antimalarial effect of natural and synthetic chalcones.

\section{Cytotoxic and Antiproliferative Activity}

Chalcones (natural and derivatives) displayed potent antiproliferative consequences in both initial as well as developed ovarian cell carcinoma (De et al., 1995) and also in stomach carcinoma HGC-27 cell (Shibata, 1994) (Table 5).

Chalcones with piperazine moiety have demonstrated different, as well as, crucial pharmacological activities counting antihistamine (Rahaman et al., 2010), antioxidant, anti-inflammatory (Bandgar and Gawande, 2010), anti-infective (Tomar et al., 2007), and anticarcinogenic properties (Filosa et al., 2007). In the light of piperazine moiety, biological activity has also been reported and encouraged.
Chalcones with piperazine moiety were created, and their in vitro anti-carcinoma-producing activity was observed (Rahaman et al., 2010). New fragrant chalcones with in vitro anti-carcinoma-producing property have also been recorded (Viveka et al., 2014). In addition, Jurkat cell line of human T-lymphocyte blood cancer along with HL-60 human blood cancer cell lines is also targeted by diaryl chalcones. The in vitro study was performed for ascertaining compound activity in opposition to two breast carcinoma cell lines MCF7 (Chauhan et al., 2014) and T47D (Jeon et al., 2016). Table 6 The result specified that all the compounds were dynamic but not analogous with doxorubicin. However, it displayed some effects against two breast carcinoma cell lines (Ugwu et al., 2015). In another study, 25 chalcone-derived compounds were reported to exhibit anticarcinogenic properties (Syam et al., 2012). Recent research conducted on 46 different chalcones to measure exact antiproliferative activities against the human tumor necrosis factor-associated programmed cell death-inducing ligand (TRAIL) against cervical (HeLa), liver (HepG2), breast (MCF7, MDA-MB-231), ovarian (Caov-3), nasopharyngeal (CNE-1), erythromyeloblastoid (K-562), lung (A549), colorectal (HT-29), T-lymphoblastoid carcinoma cells (CEM-SS), and common human embryonic kidney (HEK-293) cells.

Chalcone derivatives with enone and thiophene rings also possess activity against tubulin assembly and colchicines; they bind to tubulin of K562 cells (chronic myeloid leukemia; CML) and inhibit their growth on $\mathrm{G}_{2} / \mathrm{M}$ stage of the cell cycle (Romagnoli, 2008). In addition, those thiophene chalcone derivatives inhibit human T-lymphocyte (Molt 4 and CEM) and human cervix cancer (HeLa) cells. This research was conducted on murine blood carcinoma (L1210), murine mammary cancer (FM3A), human HeLa, Molt 4, and CEM cells by taking $0.3-0.5$ million cells $/ \mathrm{mL}$ of culture medium. After incubating the cells with testing compounds at $37^{\circ} \mathrm{C}$ for 2 days, cell number was counted by means of a Coulter counter.

\section{Anticancer Potential of Chalcones}

Cancer is one of the most feared diseases of the 21st century-according to the 2012 Globocan report, 14 million people are diagnosed with cancer each year and more than 8 million deaths are reported each year (Ferlay et al., 2013). Because radiotherapy or chemotherapy has multiple adverse effects, new molecular therapies are being tested for use in the 
TABLE 6 | In vitro summarization of recent research on heteroaromatic chalcones (Jeon et al., 2016).

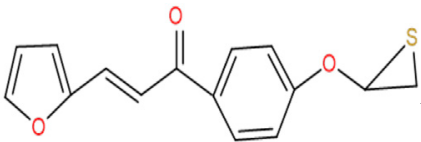

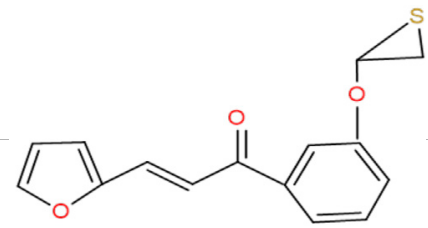

Chalcone derivate $\mathbf{D}$

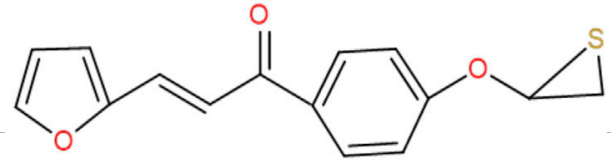

Chalcone derivate $\mathrm{E}$

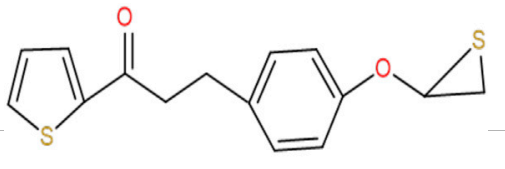

Chalcone derivate $\mathrm{F}$

Tested cells lines

MDA-MB231 basal resembling (more invasive) human triple negative breast adenocarcinoma cell line

MDA-MB468 human triple negative breast adenocarcinoma cell line originated from metastatic spot

T47D human breast ductal cancer cell line

\section{Results}

$I \mathrm{C}_{50}=\left.100 \mu \mathrm{M} \downarrow T\right|^{1}>60 \%$

$\downarrow T \|^{2}>90 \%$

$\downarrow T 47 \mathrm{D}$ carcinoma cells proliferation
$\left|\mathrm{C}_{50}=100 \mu \mathrm{M} \downarrow \mathrm{TI}\right|^{1}>70 \%$

$\downarrow T \|^{2}>90 \%$

Lactivity against all cell lines comparing

with others three chalcones
$\left|\mathrm{C}_{50}=100 \mu \mathrm{M} \downarrow T\right|^{1}>60 \%$

$\downarrow T \|^{2}>90 \%$

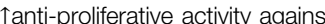

MDA-MB468
$|\mathrm{C} 50=100 \mu \mathrm{M} \downarrow \mathrm{T}| 1<5 \%$

$0 \%$ inhibition of TII

IT47D carcinoma cell proliferation IC50 $=3.85 \mu \mathrm{M}$

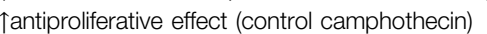


treatment of solid tumors and blood cancers (Ahmad Farooqi et al., 2017; Salehi et al., 2019e). Targeted molecular therapy uses the patient's genetic information to determine which molecules can act most effectively in concerning to the type of diagnosed cancer (Zajac et al., 2016; Žiberna et al., 2017; Moosavi et al., 2018). Thus, heat-shock proteins 90 (HSP90) inhibitors open new perspectives in cancer treatment by destabilizing proteins by which cancer cells survive and multiply (tumorgenesis) (Amolins and Blagg, 2009). Recent studies from last years have shown that synthetic chalcones can have a HSP90 inhibitory effect (Jeong et al., 2014; Oh and Seo, 2017). Several phase II clinical trials of new anticancer molecules that have two hydroxyl groups at positions 1,3 revealed inhibition of interactions between HSP90 and patients' proteins through binding of these molecules to the ATP site in HSP90 (Butler et al., 2015). Maybe in the future, phase III clinical trials will be conducted to support the anticancer potential of chalcones and their derivatives.

\section{Neuroprotective Activity}

A research has been conducted on 10 different chalcones, out of which two have nearly similar activity as of diazepam: isoliquiritigenin (ISL, 2', 4',4-trihydroxychalcone) and butein (BUT, 2', ', 3,4,tetrahydroxychalcone). This research based on outcomes of chalcones on different replacements, investigated in animal models for instance open field experiment, equine protozoal myeloencephalitis test, rotarod performance, and grip analysis. These experiments are typical models for screening CNS actions giving information regarding tranquilizing or sleep inducing, psychomotor performance, anxiety, and musclerelaxant effects (Tsatsakis A. M. et al., 2019). The kinetic study of ISL to monoamine oxidase-A indicated that it merged to variable positions of the enzyme, independent of the pre-binding of serotonin (Tan et al., 2000). In the wideranging perception, reasonably lipophilic medicines traverse the blood-brain barrier (BBB) by submissive diffusion (Salehi et al., 2020c; Sharifi-Rad et al., 2020d). Opposing molecules are usually poor central nervous system agents, except they pass through dynamic transport across the central nervous system (Pajouhesh and Lenz, 2005; Salehi et al., 2020a). Hence, it can be approximated that they are able to traverse the $\mathrm{BBB}$ and attain their target (Di et al., 2003; Calina et al., 2020; Sharifi-Rad et al., 2020e). Chalcones one, nine, fourteen, fifteen, and sixteen with fine affinity for the BZD binding positions of the GABA category A receptors, chalcones one and five with attraction for the 5hydroxytryptamine ${ }_{1 \mathrm{~A}}$ receptor, and compounds six and twelve for the $\mu$-opioid receptor were preferred to be experimented as antidepressants, anti-anxiety agents, and against the sensation and perception of pain in extensively applied pharmacological experiments in rats (Salehi et al., 2019c). During the tail suspension experiment, chalcone one demonstrated antidepressant-like activity in rodents, while compound six demonstrated action against sensations and perceptions of pain in an acute chemical stimulated nociception assessment.

The new fifty-methyl-twenty-hydroxy-thirty-nitrochalcone exhibited marginal and central activities against perceptions and sensations of pain either in acute thermal or chemical nociception experiments. According to the consequences recapitulated, plain chalcone derived compounds are favorable compounds for the discovery and growth of new central nervous system medicines and contain an encouraging scaffold in medical chemistry for the evolution of medicines and for the management of pain, depression, and anxiety (Dominguez et al., 2009).

\section{CHALCONES IN CLINICAL TRIALS Chalcones in Treatment of Chronic Venous Insufficiency}

Chronic venous insufficiency (CVI) is a clinical syndrome that results from chronic disorders of venous circulation from the lower limb level. The main symptoms in moderate stages are heavy legs, tension in the lower limbs, varicose veins dilated, followed in severe stages by swelling of the lower limbs, skin changes, and the appearance of venous ulcer (Lichota et al., 2019). A therapeutic option is represented by laser therapy, sclerotherapy, and venoactive drugs (Ianosi et al., 2019). These venoactive drugs are a heterogeneous group of substances from plant or synthetic origin that modulate the venous tone, attenuates the blood rheology, improves micro- and macrocirculation, regulates capillary permeability, have antiinflammatory effects by inhibiting leukocyte-endothelial interaction, and reduces the oxidative stress (Salehi et al., 2020b).

Recent clinical trials have shown the main role of two chalcones hesperidin methylchalcone and hesperidin trimethylchalcone in the treatment of chronic venous disorders (Boyle et al., 2003) and varices of the trunk of the internal saphenous vein, respectively (Weindorf and SchultzEhrenburg, 1987). In a randomized open-label study, the therapeutic effect of a mixture of hesperidin methyl chalcone, Ruscus aculeatus with vitamin C compared to rutozide in patients diagnosed with chronic venous insufficiency was investigated (Beltramino et al., 2000). This clinical trial was conducted for three months and included eighty patients divided into two groups: the first group received the combination with hesperidin methyl chalcone, and the second received only rutoside. The signs and symptoms of chronic venous insufficiency were evaluated initially and then monthly. From the clinical point of view, a significant and lasting reduction of the symptoms was obtained in the patients from the first group treated with the mixture of chalcone and vitamin $\mathrm{C}$ compared to the second group, treated only with rutozide (Beltramino et al., 1999).

The mechanism of the venotonic effect of Ruscus and hesperidin methylchalcone extract is exerted by a two-way adrenergic mechanism: 1) direct effect as agonist of the postjunctional alpha-adrenergic receptors of the smooth cell in the vascular wall and 2) indirect effect expressed by increasing the release of noradrenaline from the presynaptic vesicles (Beltramino et al., 1999; Peralta et al., 2007; Gomes et al., 2017). The dose-effect relationship in the single dose and the respective role of each constituent of this combination with hesperidin methylchalcone (150 mg), Ruscus aculeatus plant (150 $\mathrm{mg}$ per capsule), and ascorbic acid $(100 \mathrm{mg})$ on the 
venous tone were also demonstrated in a clinical study that included 37 women with superficial venous insufficiency. It has been shown that the effect of a capsule administered twice daily is similar to the administration of two capsules daily in the morning, and no adverse digestive effects have been reported. Clinical efficiency consisted in improving the permeability of the vascular walls, increasing the vascular tone, reducing the edema and normalizing the blood circulation in the blood vessels (Boccalon et al., 1998). Similar beneficial effect of hesperidin methylchalcone (HMC) on lymphatic venous insufficiency in a recent meta-analysis of some clinical trials has also been demonstrated. The good tolerability and the reduced adverse effects of the combination of HMC, Ruscus extract, and vitamin C have led the specialists to propose their inclusion in the new treatment guidelines for chronic venous insufficiency (Kakkos et al., 2018).

In a randomized double-blind study, the pharmacological effect of trimethyl hesperidine chalcone associated with Ruscus extract and vitamin $\mathrm{C}$ was demonstrated in patients diagnosed with femoral trunk varicose (Weindorf and Schultz-Ehrenburg, 1987). The study included fifty patients, divided into two groups: one orally treated 14 days with this combination and the other with placebo. In both the groups, the venous tone was evaluated by plethysmography, both in motion and at rest. In the group treated with trimethyl hesperidine chalcone associated with Ruscus extract and vitamin C, the clinical signs were significantly reduced (Weindorf and Schultz-Ehrenburg, 1987).

\section{Chalcones in Treatment of Skin Conditions}

Skin diseases are leading causes of morbidity with high prevalence and incidence, affecting the patients'quality of life and being associated with very important social, economic, and healthcare costs (Ianosi et al., 2018; Scheau et al., 2020). This is why the search for new treatment options in dermatology is one of the most important research areas in both fundamental and clinical science (Ianoşi et al., 2016; Sifaki et al., 2020).

Various clinical trials have evaluated the role of chalcones in inflammatory skin conditions and one of the most investigated substances was licochalcone A. An interesting study including sixty-two women with persistent mild to moderate facial redness (Weber et al., 2006) has evaluated skin compatibility and effect of a skin care regimen containing licochalcone A with duration of 8 weeks. The topical products were very well tolerated, and the results of the study showed significant improvements of erythema and in quality of life of the patients. A subsequent study on 33 rosacea patients showed that the skin care products with licochalcone A are compatible with the standard topical treatment of the disease.

Another research has assessed the effects on sensitive skin of licochalcone $\mathrm{A}$ in combination with 4-t-butylcyclohexanol (Sulzberger et al., 2016). The authors have conducted a singleblind, randomized study in order to evaluate subjective and objective symptoms of skin sensitivity. The formulation containing licochalcone A-rich licorice extract combined with 4-t-butylcyclohexanol showed a significant reduction of shavinginduced erythema. It was suggested that the anti-inflammatory effect of licochalcone $\mathrm{A}$ is induced by a significant reduction of NFKB signaling and prostaglandin E2 (PGE2) secretion.
A recent randomized, prospective, investigator-blinded study (Boonchai et al., 2018) has evaluated the effects of a moisturizer containing 4-t-butylcyclohexanol and licochalcone A on eighty patients with mild to moderate facial dermatitis. The chalcone containing topical treatment has induced significant improvements of clinical aspect, hydration of cutaneous tissue, and transepidermal water loss as well as the patients' subjective evaluation. The results of facial moisturizer were compared with those induced by $0.02 \%$ triamcinolone acetonide cream and even if the topical corticoid treatment was associated with faster improvement of patients' symptoms, the chalcone containing moisturizer showed better effects on skin hydration and inflammation control.

A complex research including two clinical studies and several in vitro experiments was conducted in order to evaluate the antiirritative effect of cosmetic formulations containing licochalcone A (Kolbe et al., 2006). The prospective randomized vehiclecontrolled clinical trials enrolled a total of 57 healthy subjects, 45 of them being included in study using a post-shaving skin irritation model and 12 volunteers taking part in a UV-induced erythema test. Even if in one model inflammation was induced by impairment of skin barrier and in the second by UV-penetration damage, in both studies, the topically applied licochalcone A-rich licorice extract showed a highly anti-irritative effect, significantly reducing erythema. The additional in vitro data emphasized possible cellular and molecular mechanisms showing a strong inhibitory effect of licochalcone A on pro-inflammatory responses of different cell types such as granulocytes, keratinocytes, dermal fibroblasts, and monocyte-derived dendritic cells.

Moreover, licochalcone A has proved to be effective in scalp disorders. The effect of a tonic solution containing licochalcone A, among other active components, has been investigated in 30 subjects with dry and itchy scalp conditions and showed a significant reduction of scalp dryness, itching, and microinflammation (Schweiger et al., 2013). The role of chalcones in the treatment of inflammatory skin conditions in children is another important area of research. A randomized, double-blind, split-side comparison study on 75 infants between the age of 2 weeks and 1 year showed that a moisturizer containing $0.025 \%$ licochalcone is equally effective as topical $1 \%$ hydrocortisone for the treatment of infantile seborrhoeic dermatitis (Wananukul et al., 2013). The same research group, in a multicenter randomized, prospective, split-side, double-blind study, has evaluated the effect of a moisturizer containing licochalcone A compared to $1 \%$ hydrocortisone topical therapy in the treatment of childhood atopic dermatitis (Wananukul et al., 2013). The study included 55 children with mild to moderate lesions and showed that the moisturizer containing licochalcone A significantly reduces the clinical severity of the lesions and the transepidermal water loss, being equally effective as topical corticosteroid treatment. Moreover, continuing the treatment with licochalcone A moisturizer was able to stabilize the clinical improvement and the skin barrier recovery. These results are in accordance with data from a previous randomized, controlled, investigator-blinded study (Udompataikul and Srisatwaja, 2011). 
Chalcones are also evaluated as potential treatment options in acne patients. A double-blinded, prospective, randomized, vehicle-controlled clinical trial has investigated the tolerability and effect of a moisturizer containing licochalcone A, L-carnitine, and 1,2-decanediol as adjuvant treatment in topical therapy with retinoids (Chularojanamontri et al., 2016). The study included 120 subjects with mild to moderate acne and showed a significant reduction of total lesions in patients treated with the moisturizer containing active substances. Moreover, they had less inflammatory lesions and skin irritations.

Anti-aging medicine is another important area of research in which chalcones are investigated (Sharifi-Rad et al., 2020b). A double-blind, placebo-controlled trial including ninety-two subjects showed that oral intake of Boesenbergia pandurata extract containing panduratin A as bioactive compound for 12 weeks significantly increases skin hydration and gloss and decreases wrinkling without any adverse symptoms, suggesting a possible use of Boesenbergia pandurata extract as a nutraceutical or nutricosmetic product (Kim et al., 2017).

\section{BIOAVAILABILITY OF CHALCONES}

Research on the bioaccessibility of chalcones from sources of food are bounded, but experimented artificial chalcones have accounted to contain broad ranges of biological activities (Won et al., 2005). Although chalcones have an essential position in the bio-production of flavonoids (Shirley, 1996) and are familiar in a number of foods and drinks, like rooibos tea or apples, there are unavailability of data on their bioaccessibility in human beings.

The prenylated chalcone xanthohumol is the amplest chalcone produced in hop cones. Throughout beer preparation, a huge fraction of xanthohumol is changed to the related isomeric prenylflavanone isoxanthohumol. Following administration of xanthohumol to rodents by force feeding at extremely elevated dosage ( $1 \mathrm{~g} / \mathrm{kg}$ of body weight), linked metabolites were identified in plasma. The most important metabolite, xanthohumol- 49-Oglucuronide, attained its topmost concentration of $3.1 \mathrm{lmol} / \mathrm{L} 4 \mathrm{~h}$ after administration. The maximum concentration of unmetabolized xanthohumol was 10 times lower with the similar Tmax of $4 \mathrm{~h}$ (Gerhäuser, 2005). One more rodent study discovered only conjugates in plasma following oral administration of xanthohumol however unsuccessful to distinguish unmetabolized xanthohumol (Avula et al., 2004).

Conversely, these studies demonstrate that prenylated chalcones are bioavailable, although their bioaccessibility appears to be commonly low.

Another study explored the prospective accessibility of flavanones in diversely processed Citrus sinensis (L.) Osbeck juices by imitating stomach and small intestinal in vitro digestion (Gil-Izquierdo et al., 2001).

In addition to showing the power of pasteurization and storage on the substance of dissolvable flavanones, these researchers detected that in vitro pancreatin intake of Citrus sinensis (L.) Osbeck juice in a mild alkaline medium, imitating absorption in the small intestine, converted fifty to sixty\% of the dissolved flavanones (primarily hesperidin) to chalcones (principally hesperidin chalcone) (Cermak et al., 2009). Particularly the poor dissolvability of a large number of chalcone compounds, the bioequivalence effectiveness has not achieved the anticipated intensities in preclinical assessments.

Therefore, the maximization of the physicochemical activities will be one of the principal study routes of chalcone-dependent compounds. For the objects of chalcone compounds, a number of anticipated targets must be confirmed. Activity-dependent protein outlining is a potent approach for recognition of target that must be decided by considering each case individually because of the properties of chalcone molecules (Zhuang et al., 2017).

\section{DISCUSSION}

The results of our study confirmed the therapeutic potential of chalcones. The limitations of this research result from the fact that many meta-analyzes were included and not individual studies. But this can be considered as a strong point because recent meta-analyzes have summarized the most important pharmacological effects in vitro and especially in vivo. Another strength of this review is that the latest studies and clinical trials on patients have been described, thus confirming the clinical importance and positive prospects in medical therapy.

Natural and synthetic chalcones and their derivatives presented antidiabetic effects, and the effect can be attributed mainly to lowering of insulin secretion with potency similar to that of hypoglycemic agents (ig Glipizide) (Jamal et al., 2009). Numerous studies have reported the anti-inflammatory effects of chalcones on several targets such as enzymes implication in promoting inflammation process: cyclo-oxygenase, interleukins, nitric oxide synthase, cell adhesion molecules (CAM), lipooxygenase (LOX), and prostaglandins (PGs) (Salehi et al., 2020c; Mititelu et al., 2020). The suppression and/or inhibition of cyclooxygenase enzyme is a promising therapeutic way in the treatment of inflammatory diseases (Salehi et al., 2019d; Sharifi-Rad et al., 2020c). Many bioactive compounds, both natural and synthetic, have been isolated and synthetized to develop anti-cyclooxygenase activity (Salehi et al., 2019b; Padureanu et al., 2019; Sharifi-Rad et al., 2020a). PGE2 and NO are among the inflammatory mediators that promote inflammation in several diseases (Salehi et al., 2019c; Salehi et al., 2020b). Consequently, the inhibition of these mediators is strongly suggested as remedy for numerous inflammatory diseases. (Mocan et al., 2014; Tsatsakis A. et al., 2019; Toiu et al., 2019). Chalcones also have proved their ability to inhibit NF- $\kappa \mathrm{B}$ (nuclear factor kappa) which regulates the most important factors involved in inflammatory process such as cytokines, chemokines, and adhesion molecules (Salehi et al., 2019d; Salehi et al., 2019a). Several studies have suggested the use of chalcones and their derivatives target specifically NF- $\kappa \mathrm{B}$ as an antiinflammatory therapeutic strategy (Chu and Guo, 2016).

Chalcones are natural products, produced by plants as a natural defense mechanism against pathogens as fungi and bacteria. Synthesized $\beta$-chlorovinyl chalcones exhibited antifungal activity (Bandgar and Gawande, 2010). In general, 
the natural chalcones (synthesized or modified) are being increasingly documented because of their interesting antimicrobial activities and can be represented as promising agents in the perspective of new antibiotic drugs discovery. Some of the chalcones have been implicated in inhibition of exoenzymes responsible for fungal invasion mechanisms, also inhibiting biofilm and germ tube formation as in C. albicans. They may affect the cellular cytoplasmic membrane and induce cell apoptosis as it was noted in case of carvacrol (Zuzarte et al., 2012). In addition, it was also reported that flavonoid compounds as chalcones inhibit the growth of bacteria by acting on the membrane potential which might affect the overall bacterial metabolic activity, resulting in some biosynthetic pathway inhibition, as demonstrated by the strong inhibition of DNA, RNA, and protein synthesis (Dzoyem et al., 2013; Ungureanu et al., 2017). Chalcones also showed to be a promising anticancer potential because it induces selective cell death in carcinoma cells with not upsetting regular cells (Syam et al., 2012) and psychoactive and neuroprotective activities. (Brady et al., 2012).

\section{OVERALL CONCLUSIONS AND FUTURE PERSPECTIVES}

The curiosity and attraction toward natural compounds are increasing gradually because of the recognized favorable consequences on numerous prevalent and general diseases like carcinoma, allergic reactions, cardiovascular disease, infectious diseases, parasitic diseases, type 2 diabetes mellitus, or diseases of central nervous system. Starting from the ethnopharmacological uses of chalcones, in this study, the most important in vitro and in vivo biological activities such as antibacterial, antioxidant, antineoplastic, cytotoxic, antiulcer, antidepressant, anxiolytic, and anti-inflammatory were highlighted. Chalcones derivatives have shown anticancer activity against a variety of cancer cell lines, antibacterial activity against Gram-negative and Grampositive germs, and anti protozoal activity. Although conducted in a small number, clinical studies of chalcones have shown a lack of adverse effects in patients with chronic venous insufficiency, the

\section{REFERENCES}

Abbas, A., Naseer, M. M., Hasan, A., and Hadda, T. B. (2014). Synthesis and cytotoxicity studies of 4-alkoxychalcones as new antitumor agents. J. Mater. Environ. Sci. 5, 281-292.

Acharjee, S., Maity, T. K., Samanta, S., Mana, S., Chakraborty, T., Singha, T., et al. (2018). Antihyperglycemic activity of chalcone based novel 1-\{3-[3-(substituted phenyl) prop-2-enoyl] phenyl\} thioureas. Synth. Commun. 48, 3015-3024. doi:10.1080/00397911.2018.1539178

Ahmad Farooqi, A., Fayyaz, S., Silva, A. S., Sureda, A., Nabavi, S. F., Mocan, A., et al. (2017). Oleuropein and cancer chemoprevention: the link is hot. Molecules 22, 705. doi:10.3390/molecules 22050705

Ajaiyeoba, E., Ogbole, O., Abiodun, O., Ashidi, J., Houghton, P., and Wright, C. W. (2013). Cajachalcone: an antimalarial compound from cajanus cajan leaf extract. J. Parasitol. Res. 2013, 703781. doi:10.1155/2013/703781

Alberton, E. H., Damazio, R. G., Cazarolli, L. H., Chiaradia, L. D., Leal, P. C., Nunes, R. J., et al. (2008). Influence of chalcone analogues on serum glucose reduction of clinical signs and symptoms, and good plasma concentrations. However, further clinical studies are needed to fully understand the mechanisms of action at the cellular level and to establish correlations between their structure and pharmacological actions, especially anticancer activity.

Although they showed many interesting biological effects and many preclinical experiments could be performed, their mechanism of action is not entirely known. Being compounds that could be synthesized relatively easily, in the future, it is necessary to develop new synthesis methods that allow the research of new biological properties, a deeper knowledge of the molecular mechanisms of action, and especially the identification of the target of the action. And so, this successful story of the promising therapeutic effects of chalcones to be applicable in the discovery of new drugs, pharmaceutical forms, using modern strategies, especially new nano-formulations in order to increase their bioavailability, prolonged effect, or transport to the target of the action. Further research and clinical trials can explore its pharmacological actions, their interactions with other compounds or medicines, and the level of toxicity it can cause.

\section{AUTHOR CONTRIBUTIONS}

JS-R, MM, and DC: conceptualization. BS, IC, NE, ABa, ABo, $\mathrm{MA}$, and MI: validation investigation. CQ, JS-R, CC, $\mathrm{AD}$, and MM: resources. CQ, JS-R, CC, GL-G, AD, MM, and FL: data curation. JS-R, AD, MM, DC, VL, and FL: review and editing. All authors: writing. All authors read and approved the final version, and contributed equally to the manuscript.

\section{FUNDING}

This research and article processing charges were funded by a grant of Romanian Ministry of Research and Innovation, CCCDI-UEFISCDI [project number 61PCCDI/2018 PN-IIIP1-1.2-PCCDI-2017-0341], within PNCDI-III.

levels in hyperglycemic rats. Chem. Biol. Interact. 171, 355-362. doi:10.1016/j. cbi.2007.11.001

Amole, K. L., Bello, I. A., and Oyewale, A. O. (2019). Synthesis, characterization and antibacterial activities of new fluorinated chalcones. Chem. Afr. 2, 47-55. doi:10.1007/s42250-019-00043-4

Amolins, M. W., and Blagg, B. (2009). Natural product inhibitors of Hsp90: potential leads for drug discovery. Mini Rev. Med. Chem. 9, 140-152. doi:10. 2174/138955709787316056

Andrighetti-Fröhner, C. R., De Oliveira, K. N., Gaspar-Silva, D., Pacheco, L. K., Joussef, A. C., Steindel, M., et al. (2009). Synthesis, biological evaluation and SAR of sulfonamide 4-methoxychalcone derivatives with potential antileishmanial activity. Eur. J. Med. Chem. 44, 755-763. doi:10.1016/j. ejmech.2008.04.016

Ansari, F. L., Umbreen, S., Hussain, L., Makhmoor, T., Nawaz, S. A., Lodhi, M. A., et al. (2005). Syntheses and biological activities of chalcone and 1, 5benzothiazepine derivatives: promising new free-radical scavengers, and esterase, urease, and a-glucosidase inhibitors. Chem. Biodivers. 2, 487-496. doi:10.1002/cbdv.200590029 
Araico, A., Terencio, M., Alcaraz, M., Dominguez, J., Leon, C., and Ferrandiz, M. (2006). Phenylsulphonyl urenyl chalcone derivatives as dual inhibitors of cyclo-oxygenase-2 and 5-lipoxygenase. Life Sci. 78, 2911-2918. doi:10.1016/j.lff.2005.11.017

Asogan, M. V., and Aupati, V. R. (2016). Discovery of synthetic bioactive flavonoid derivatives as potential antidiabetic agents. Der Pharma Chem. 8, 152-168.

Attarde, M., Vora, A., Varghese, A., and Kachwala, Y. (2014). Synthesis and evaluation of chalcone derivatives for its alpha amylase inhibitory activity. Org. Chem. An Indian J. 10, 192-204.

Avula, B., Ganzera, M., Warnick, J. E., Feltenstein, M. W., Sufka, K. J., and Khan, I. A. (2004). High-performance liquid chromatographic determination of xanthohumol in rat plasma, urine, and fecal samples. J. Chromatogr. Sci. 42, 378-382. doi:10.1093/chromsci/42.7.378

Bak, E. J., Park, H. G., Lee, C., Lee, T.-I., Woo, G.-H., Na, Y., et al. (2011). Effects of novel chalcone derivatives on a-glucosidase, dipeptidyl peptidase-4, and adipocyte differentiation in vitro. BMB Rep. 44, 410-414. doi:10.5483/ BMBRep.2011.44.6.410

Bandgar, B. P., and Gawande, S. S. (2010). Synthesis and biological screening of a combinatorial library of $\beta$-chlorovinyl chalcones as anticancer, antiinflammatory and antimicrobial agents. Bioorg. Med. Chem. 18, 2060-2065. doi:10.1016/j.bmc.2009.12.077

Bandgar, B. P., Patil, S. A., Gacche, R. N., Korbad, B. L., Hote, B. S., Kinkar, S. N., et al. (2010). Synthesis and biological evaluation of nitrogen-containing chalcones as possible anti-inflammatory and antioxidant agents. Bioorg. Med. Chem. Lett. 20, 730-733. doi:10.1016/j.bmcl.2009.11.068

Bano, S., Javed, K., Ahmad, S., Rathish, I., Singh, S., Chaitanya, M., et al. (2013). Synthesis of some novel chalcones, flavanones and flavones and evaluation of their anti-inflammatory activity. Eur. J. Med. Chem. 65, 51-59. doi:10.1016/j. ejmech.2013.04.056

Batovska, D., Slavova, A., Bankova, V., Tsvetkova, I., Ninova, M., and Najdenski, H. (2007). Study on the substituents' effects of a series of synthetic chalcones against the yeast candida albicans. Eur. J. Med. Chem. 42, 87-92. doi:10.1016/j. ejmech.2006.08.012

Bello, M. L., Chiaradia, L. D., Dias, L. R. S., Pacheco, L. K., Stumpf, T. R., Mascarello, A., et al. (2011). Trimethoxy-chalcone derivatives inhibit growth of Leishmania braziliensis: synthesis, biological evaluation, molecular modeling and structure-activity relationship (SAR). Bioorg. Med. Chem. 19, 5046-5052. doi:10.1016/j.bmc.2011.06.023

Beltramino, R. P., Penenory, A., and Buceta, A. M. (1999). An open-label, randomised multicentre study comparing the efficacy and safety of CYCLO 3 FORT versus hydroxyethyl rutoside in chronic venous lymphatic insufficiency. Int. Angiol. 18, 337-342.

Beltramino, R., Penenory, A., and Buceta, A. M. (2000). An open-label, randomized multicenter study comparing the efficacy and safety of Cyclo 3 Fort $^{\circledR}$ versus hydroxyethyl rutoside in chronic venous lymphatic insufficiency. Angiology 51, 535-544. doi:10.1177/000331970005100702

Bhakuni, D., and Chaturvedi, R. (1984). Chemical constituents of Crotalaria madurensis. J. Nat. Prod. 47, 585-591. doi:10.1021/np50034a003

Bhale, P. S., Dongare, S. B., and Chanshetti, U. B. (2013). Synthesis and antimicrobial screening of chalcones containing imidazo $[1,2-\mathrm{a}]$ pyridine nucleus. Res. J. Chem. Sci. 2231, 606.

Bharatham, K., Bharatham, N., Park, K. H., and Lee, K. W. (2008). Binding mode analyses and pharmacophore model development for sulfonamide chalcone derivatives, a new class of a-glucosidase inhibitors. J. Mol. Graph. Model. 26, 1202-1212. doi:10.1016/j.jmgm.2007.11.002

Boccalon, H., Causse, C., and Yubero, L. (1998). Comparative efficacy of a single daily dose of two capsules Cyclo 3 Fort in the morning versus a repeated dose of one capsule morning and noon: a one-month study. Int. Angiol. 17, 155.

Boonchai, W., Varothai, S., Winayanuwattikun, W., Phaitoonvatanakij, S., Chaweekulrat, P., and Kasemsarn, P. (2018). Randomized investigatorblinded comparative study of moisturizer containing 4-t-butylcyclohexanol and licochalcone $\mathrm{A}$ versus $0.02 \%$ triamcinolone acetonide cream in facial dermatitis. J. Cosmet. Dermatol. 17, 1130-1135. doi:10.1111/jocd.12499

Borges-Argaez, R., Balnbury, L., Flowers, A., Giménez-Turba, A., Ruiz, G., Waterman, P. G., et al. (2007). Cytotoxic and antiprotozoal activity of flavonoids from Lonchocarpus spp. Phytomedicine 14, 530-533. doi:10.1016/ j.phymed.2006.11.027

Boyle, P., Diehm, C., and Robertson, C. (2003). Meta-analysis of clinical trials of cyclo 3 Fort in the treatment of chronic venous insufficiency. Int. Angiol. 22, 250.
Brady, S., Siegel, G., Albers, R. W., and Price, D. (2012). Basic Neurochemistry: Principles of Molecular, Cellular, and Medical Neurobiology. Elsevier Academic Press. Available at: https://doi.org/10.1016/C2009-0-00066-X

Burmaoglu, S., Yilmaz, A. O., Polat, M. F., Kaya, R., Gulcin, I., and Algul, O. (2019). Synthesis and biological evaluation of novel tris-chalcones as potent carbonic anhydrase, acetylcholinesterase, butyrylcholinesterase and $\alpha$-glycosidase inhibitors. Bioorg. Chem. 85, 191-197. doi:10.1016/j.bioorg.2018.12.035

Butler, L. M., Ferraldeschi, R., Armstrong, H. K., Centenera, M. M., and Workman, P. (2015). Maximizing the therapeutic potential of HSP90 inhibitors. Mol. Cancer Res. 13, 1445-1451. doi:10.1158/1541-7786.MCR-15-0234

Cai, C.-Y., Rao, L., Rao, Y., Guo, J.-X., Xiao, Z.-Z., Cao, J.-Y., et al. (2017). Analogues of xanthones-chalcones and bis-chalcones as $\alpha$-glucosidase inhibitors and anti-diabetes candidates. Eur. J. Med. Chem. 130, 51-59. doi:10.1016/j.ejmech.2017.02.007

Calina, D., Buga, A. M., Mitroi, M., Buha, A., Caruntu, C., Scheau, C., et al. (2020). The treatment of cognitive, behavioural and motor impairments from brain injury and neurodegenerative diseases through cannabinoid system modulation-evidence from in vivo studies. J. Clin. Med. 9 (8), 2395. doi:10. $3390 / \mathrm{jcm} 9082395$

Cermak, R., Durazzo, A., Maiani, G., Böhm, V., Kammerer, D. R., Carle, R., et al. (2009). The influence of postharvest processing and storage of foodstuffs on the bioavailability of flavonoids and phenolic acids. Mol. Nutr. Food Res. 53, S184-S193. doi:10.1002/mnfr.200700444

Chatsumpun, N., Sritularak, B., and Likhitwitayawuid, K. (2017). New biflavonoids with $a$-glucosidase and pancreatic lipase inhibitory activities from boesenbergia rotunda. Molecules 22, 1862. doi:10.3390/molecules 22111862

Chatzopoulou, M., Pegklidou, K., Papastavrou, N., and Demopoulos, V. J. (2013). Development of aldose reductase inhibitors for the treatment of inflammatory disorders. Expet Opin. Drug Discov. 8, 1365-1380. doi:10.1517/17460441.2013.843524

Chauhan, S. S., Singh, A. K., Meena, S., Lohani, M., Singh, A., Arya, R. K., et al. (2014). Synthesis of novel $\beta$-carboline based chalcones with high cytotoxic activity against breast cancer cells. Bioorg. Med. Chem. Lett. 24, 2820-2824. doi:10.1016/j.bmcl.2014.04.109

Chen, J.-J., Cheng, M.-J., Shu, C.-W., Sung, P.-J., Lim, Y.-P., Cheng, L.-Y., et al. (2017). A new chalcone and antioxidant constituents of Glycyrrhiza glabra. Chem. Nat. Compd. 53, 632-634. doi:10.1007/s10600-017-2077-1

Chen, M., Christensen, S. B., Blom, J., Lemmich, E., Nadelmann, L., Fich, K., et al. (1993). Licochalcone A, a novel antiparasitic agent with potent activity against human pathogenic protozoan species of leishmania. Antimicrob. Agents Chemother. 37, 2550-2556. doi:10.1128/aac.37.12.2550

Chen, M., Christensen, S., Theander, T. G., and Kharazmi, A. (1994). Antileishmanial activity of licochalcone A in mice infected with leishmania major and in hamsters infected with Leishmania donovani. Antimicrob. Agents Chemother. 38, 1339-1344. doi:10.1128/aac.38.6.1339

Chinthala, Y., Thakur, S., Tirunagari, S., Chinde, S., Domatti, A. K., Arigari, N. K., et al. (2015). Synthesis, docking and ADMET studies of novel chalcone triazoles for anti-cancer and anti-diabetic activity. Eur. J. Med. Chem. 93, 564-573. doi:10.1016/j.ejmech.2015.02.027

Chu, J., and Guo, C. L. (2016). Design and discovery of some novel chalcones as antioxidant and anti-inflammatory agents via attenuating NF-kB. Arch. Pharm. (Weinheim) 349, 63-70. doi:10.1002/ardp.201500349

Chularojanamontri, L., Tuchinda, P., Kulthanan, K., Varothai, S., and Winayanuwattikun, W. (2016). A double-blinded, randomized, vehiclecontrolled study to access skin tolerability and efficacy of an antiinflammatory moisturizer in treatment of acne with $0.1 \%$ adapalene gel. J. Dermatolog. Treat. 27, 140-145. doi:10.3109/09546634.2015. 1079298

Daikonya, A., Katsuki, S., and Kitanaka, S. (2004). Antiallergic agents from natural sources 9 . Inhibition of nitric oxide production by novel chalcone derivatives from Mallotus philippinensis (Euphorbiaceae). Chem. Pharm. Bull. (Tokyo) 52, 1326-1329. doi:10.1248/cpb.52.1326

Dal Picolo, C. R., Bezerra, M. P., Gomes, K. S., Passero, L. F. D., Laurenti, M. D. Martins, E. G. A., et al. (2014). Antileishmanial activity evaluation of adunchalcone, a new prenylated dihydrochalcone from Piper aduncum L. Fitoterapia 97, 28-33. doi:10.1016/j.fitote.2014.05.009

Damazio, R. G., Zanatta, A. P., Cazarolli, L. H., Chiaradia, L. D., Mascarello, A., Nunes, R. J., et al. (2010). Antihyperglycemic activity of naphthylchalcones. Eur. J. Med. Chem. 45, 1332-1337. doi:10.1016/j.ejmech.2009.12.017 
Damazio, R. G., Zanatta, A. P., Cazarolli, L. H., Mascarello, A., Chiaradia, L. D., Nunes, R. J., et al. (2009). Nitrochalcones: potential in vivo insulin secretagogues. Biochimie 91, 1493-1498. doi:10.1016/j.biochi.2009.09.002

De Mello, T. F., Bitencourt, H. R., Pedroso, R. B., Aristides, S. M., Lonardoni, M. V., and Silveira, T. G. (2014). Leishmanicidal activity of synthetic chalcones in Leishmania (Viannia) braziliensis. Exp. Parasitol. 136, 27-34. doi:10.1016/j. exppara.2013.11.003

De, R. V., Scambia, G., Benedetti, P. P., Ranelletti, F., Bonanno, G., Ercoli, A., et al. (1995). Effect of synthetic and naturally occurring chalcones on ovarian cancer cell growth: structure-activity relationships. Anticancer Drug Des. 10, 481-490.

Di, L., Kerns, E. H., Fan, K., Mcconnell, O. J., and Carter, G. T. (2003). High throughput artificial membrane permeability assay for blood-brain barrier. Eur. J. Med. Chem. 38, 223-232. doi:10.1016/s0223-5234(03)00012-6

Domínguez, J. N., León, C., Rodrigues, J., De Domínguez, N. G., Gut, J., and Rosenthal, P. J. (2005). Synthesis and antimalarial activity of sulfonamide chalcone derivatives. Farmaco 60, 307-311. doi:10.1016/j.farmac.2005.01. 005

Dominguez, J. N., Leon, C., Rodrigues, J., De Dominguez, N. G., Gut, J., and Rosenthal, P. J. (2009). Synthesis of chlorovinyl sulfones as structural analogs of chalcones and their antiplasmodial activities. Eur. J. Med. Chem. 44, 1457-1462. doi:10.1016/j.ejmech.2008.09.044

Domínguez, J. N., Charris, J. E., Lobo, G., De Domínguez, N. G., Moreno, M. M., Riggione, F., et al. (2001). Synthesis of quinolinyl chalcones and evaluation of their antimalarial activity. Eur. J. Med. Chem. 36, 555-560. doi:10.1016/s02235234(01)01245-4

Dzoyem, J. P., Hamamoto, H., Ngameni, B., Ngadjui, B. T., and Sekimizu, K. (2013). Antimicrobial action mechanism of flavonoids from dorstenia species. Drug Discov. Ther. 7, 66-72. doi:10.5582/ddt.2013.v7.2.66

Elsohly, H., Joshi, A., Nimrod, A., Walker, L., and Clark, A. (2001). Antifungal chalcones from maclura tinctoria. Planta Med. 67, 87-89. doi:10.1055/s-200110621

Enoki, T., Ohnogi, H., Kobayashi, E., and Sagawa, H. (2010). Anti-diabetic activities of chalcones derived from ashitaba. Nippon Shokuhin Kagaku Kogaku Kaishi 57, 456-463. doi:10.3136/nskkk.57.456

Enoki, T., Ohnogi, H., Nagamine, K., Kudo, Y., Sugiyama, K., Tanabe, M., et al. (2007). Antidiabetic activities of chalcones isolated from a japanese herb, angelica keiskei. J. Agric. Food Chem. 55, 6013-6017. doi:10.1021/ jf070720q

Farzaneh, S., Zeinalzadeh, E., Daraei, B., Shahhosseini, S., and Zarghi, A. (2018). New ferrocene compounds as selective cyclooxygenase (COX-2) inhibitors: design, synthesis, cytotoxicity and enzyme-inhibitory activity. Anticancer Agents Med. Chem. 18, 295-301. doi:10.2174/1871520617666171003145533

Ferlay, J., Soerjomataram, I., Ervik, M., Dikshit, R., Eser, S., Mathers, C., Rebelo, M., Parkin, D. M., Forman, D., and Bray, F. (2013). GLOBOCAN 2012 v1.0, Cancer Incidence and Mortality Worldwide: IARC CancerBase No. 11 [Internet]. Lyon, France: International Agency for Research on Cancer. Available at: http:// globocan.iarc.fr.

Filosa, R., Peduto, A., De Caprariis, P., Saturnino, C., Festa, M., Petrella, A., et al. (2007). Synthesis and antiproliferative properties of N3/8-disubstituted 3, 8diazabicyclo [3.2. 1] octane analogues of 3, 8-bis [2-(3, 4, 5-trimethoxyphenyl) pyridin-4-yl] methyl-piperazine. Eur. J. Med. Chem. 42, 293-306. doi:10.1016/j. ejmech.2006.11.013

Frölich, S., Schubert, C., and Jenett-Siems, K. (2009). Antimalarials from prenylated chalcone derivatives of hops. Beer Health Dis. Prev., 747-752. doi:10.1016/b978-0-12-373891-2.00075-4

Gabriela, N., Rosa, A. M., Catiana, Z. I., Soledad, C., Mabel, O. R., Esteban, S. J., et al. (2014). The effect of zuccagnia punctata, an argentine medicinal plant, on virulence factors from candida species. Natural Prod. Commun. 9, 1934578X1400900712. doi:10.1177/1934578x1400900712

Gaur, R., Yadav, K. S., Verma, R. K., Yadav, N. P., and Bhakuni, R. S. (2014). In vivo anti-diabetic activity of derivatives of isoliquiritigenin and liquiritigenin. Phytomedicine 21, 415-422. doi:10.1016/j.phymed.2013.10.015

Gerhäuser, C. (2005). Beer constituents as potential cancer chemopreventive agents. Eur. J. Cancer 41, 1941-1954. doi:10.1016/j.ejca.2005.04.012

Geyer, J. A., Keenan, S. M., Woodard, C. L., Thompson, P. A., Gerena, L., Nichols, D. A., et al. (2009). Selective inhibition of Pfmrk, a plasmodium falciparum CDK, by antimalarial 1, 3-diaryl-2-propenones. Bioorg. Med. Chem. Lett. 19, 1982-1985. doi:10.1016/j.bmcl.2009.02.042
Gil-Izquierdo, A., Gil, M. I., Ferreres, F., and Tomás-Barberán, F. A. (2001). In vitro availability of flavonoids and other phenolics in orange juice. J. Agric. Food Chem. 49, 1035-1041. doi:10.1021/jf0000528

Gilmore, T. D. (2006). Introduction to NF-א B: players, pathways, perspectives. Oncogene 25, 6680-6684. doi:10.1038/sj.onc.1209954

Gomes, M. N., Muratov, E. N., Pereira, M., Peixoto, J. C., Rosseto, L. P., Cravo, P. V., et al. (2017). Chalcone derivatives: promising starting points for drug design. Molecules 22, 1210. doi:10.3390/molecules 22081210

Guantai, E. M., Ncokazi, K., Egan, T. J., Gut, J., Rosenthal, P. J., Smith, P. J., et al. (2010). Design, synthesis and in vitro antimalarial evaluation of triazole-linked chalcone and dienone hybrid compounds. Bioorg. Med. Chem. 18, 8243-8256. doi:10.1016/j.bmc.2010.10.009

Gupta, S., Shivahare, R., Korthikunta, V., Singh, R., Gupta, S., and Tadigoppula, N. (2014). Synthesis and biological evaluation of chalcones as potential antileishmanial agents. Eur. J. Med. Chem. 81, 359-366. doi:10.1016/j. ejmech.2014.05.034

Ha, M. T., Seong, S. H., Nguyen, T. D., Cho, W.-K., Ah, K. J., Ma, J. Y., et al. (2018). Chalcone derivatives from the root bark of Morus alba L. act as inhibitors of PTP1B and a-glucosidase. Phytochemistry 155, 114-125. doi:10.1016/j. phytochem.2018.08.001

Hara, H., Ikeda, R., Ninomiya, M., Kamiya, T., Koketsu, M., and Adachi, T. (2014). Newly synthesized 'hidabeni' chalcone derivatives potently suppress LPSinduced NO production via inhibition of STAT1, but not NF-kB, JNK, and p38, pathways in microglia. Biol. Pharm. Bull. 37, 1042-1049. doi:10.1248/bpb. b14-00116

Hayat, F., Moseley, E., Salahuddin, A., Van Zyl, R. L., and Azam, A. (2011). Antiprotozoal activity of chloroquinoline based chalcones. Eur. J. Med. Chem. 46, 1897-1905. doi:10.1016/j.ejmech.2011.02.004

Hermoso, A., Jiménez, I. A., Mamani, Z. A., Bazzocchi, I. L., Piñero, J. E., Ravelo, A. G., et al. (2003). Antileishmanial activities of dihydrochalcones from piper elongatum and synthetic related compounds. structural requirements for activity. Bioorg. Med. Chem. 11, 3975-3980. doi:10.1016/s0968-0896(03)00406-1

Hideo, K., and Tatsurou, H. (1997). Yoko and Kojima. Chem. Abstr. 126, 125

Huang, Y. C., Guh, J. H., Cheng, Z. J., Chang, Y. L., Hwang, T. L., Lin, C. N., et al. (2001). Inhibitory effect of DCDC on lipopolysaccharide-induced nitric oxide synthesis in RAW 264.7 cells. Life Sci. 68, 2435-2447. doi:10.1016/s00243205(01)01035-9

Ianosi, G., Ianosi, S., Calbureanu-Popescu, M. X., Tutunaru, C., Calina, D., and Neagoe, D. (2019). Comparative study in leg telangiectasias treatment with Nd: YAG laser and sclerotherapy. Exp. Ther. Med. 17, 1106-1112. doi:10.3892/etm.2018.6985

Ianoşi, S., Ianoşi, G., Neagoe, D., Ionescu, O., Zlatian, O., Docea, A. O., et al. (2016). Age-dependent endocrine disorders involved in the pathogenesis of refractory acne in women. Mol. Med. Rep. 14, 5501-5506. doi:10.3892/mmr. 2016.5924

Ianosi, S., Neagoe, D., Branisteanu, D. E., Popescu, M., Calina, D., Zlatian, O., et al. (2018). Comparative efficacy of oral contraceptive versus local treatment versus intense pulsed light combined with vacuum in endocrine acne in women. J. Biol. Regul. Homeost. Agents 32, 711-718.

Imran, S., Taha, M., Ismail, N. H., Kashif, S. M., Rahim, F., Jamil, W., et al. (2015). Synthesis of novel flavone hydrazones: in-vitro evaluation of a-glucosidase inhibition, QSAR analysis and docking studies. Eur. J. Med. Chem. 105, 156-170. doi:10.1016/j.ejmech.2015.10.017

Jadhav, S. Y., Bhosale, R. B., Shirame, S. P., Hublikar, M. G., Sonawane, K. D., Shaikh, R. V., et al. (2013). Synthesis and biological evaluation of fluorohydroxy substituted pyrazole chalcones as anti-inflammatory, antioxidant and antibacterial agents. Int. J. Pharm. Biol. Sci. 4, 309-397.

Jain, A., and Jain, D. (2017). Docking, synthesis and evaluation of novel derivatives of substituted chalcones as antihyperglycemic agents. J. Drug Deliv. Ther. 7, 154-157.

Jamal, H., Ansari, W., and Rizvi, S. (2009). Chalcones: differential effects on glycogen contents of liver, brain, and spinal cord in rats. Biol. Med. 1, 107-115.

Jantan, I., Bukhari, S. N. A., Adekoya, O. A., and Sylte, I. (2014). Studies of synthetic chalcone derivatives as potential inhibitors of secretory phospholipase A2, cyclooxygenases, lipoxygenase and pro-inflammatory cytokines. Drug Des. Devel. Ther. 8, 1405. doi:10.2147/DDDT.S67370

Jayanthi, M., Jegatheesan, K., Vidhya, R., and Kanagavalli, U. (2012). Hypoglycemic effect of 2-hydroxychalcone on high fructose fed diabetic rat. Int. J. Pharm. Sci. Res. 3, 600. 
Jayasinghe, L., Balasooriya, B., Padmini, W. C., Hara, N., and Fujimoto, Y. (2004). Geranyl chalcone derivatives with antifungal and radical scavenging properties from the leaves of artocarpus nobilis. Phytochemistry 65, 1287-1290. doi:10. 1016/j.phytochem.2004.03.033

Jeon, K.-H., Yu, H.-B., Kwak, S. Y., Kwon, Y., and Na, Y. (2016). Synthesis and topoisomerases inhibitory activity of heteroaromatic chalcones. Bioorg. Med. Chem. 24, 5921-5928. doi:10.1016/j.bmc.2016.09.051

Jeong, C.-H., Park, H. B., Jang, W. J., Jung, S. H., and Seo, Y. H. (2014). Discovery of hybrid Hsp90 inhibitors and their anti-neoplastic effects against gefitinibresistant non-small cell lung cancer (NSCLC). Bioorg. Med. Chem. Lett. 24, 224-227. doi:10.1016/j.bmcl.2013.11.034

Kakkos, S. K., Bouskela, E., Jawien, A., and Nicolaides, A. N. (2018). New data on chronic venous disease: a new place for cyclo $3^{\circledR}$ fort. Int. Angiol. 37, 85-92. doi:10.23736/S0392-9590.17.03935-9

Kim, D. H., Li, H., Han, Y. E., Jeong, J. H., Lee, H. J., and Ryu, J.-H. (2018). Modulation of inducible nitric oxide synthase expression in LPS-stimulated BV-2 microglia by prenylated chalcones from Cullen corylifolium (L.) Medik. Through inhibition of I-kBa degradation. Molecules 23, 109. doi:10.3390/ molecules 23010109

Kim, D. U., Chung, H. C., Kim, C., and Hwang, J. K. (2017). Oral intake of boesenbergia pandurata extract improves skin hydration, gloss, and wrinkling: a randomized, double-blind, and placebo-controlled study. J. Cosmet. Dermatol. 16, 512-519. doi:10.1111/jocd.12343

Kim, J. H., Ryu, Y. B., Kang, N. S., Lee, B. W., Heo, J. S., Jeong, I.-Y., et al. (2006). Glycosidase inhibitory flavonoids from sophora flavescens. Biol. Pharm. Bull. 29, 302-305. doi:10.1248/bpb.29.302

Kim, M. J., Kadayat, T., Da Eun Kim, E.-S. L., and Park, P.-H. (2014). TI-I-174, a synthetic chalcone derivative, suppresses nitric oxide production in murine macrophages via heme oxygenase-1 induction and inhibition of AP-1. Biomol. Ther. (Seoul) 22, 390. doi:10.4062/biomolther.2014.062

Kim, Y. H., Kim, J., Park, H., and Kim, H. P. (2007). Anti-inflammatory activity of the synthetic chalcone derivatives: inhibition of inducible nitric oxide synthasecatalyzed nitric oxide production from lipopolysaccharide-treated RAW 264.7 cells. Biol. Pharm. Bull. 30, 1450-1455. doi:10.1248/bpb.30.1450

Kolbe, L., Immeyer, J., Batzer, J., Wensorra, U., Tom Dieck, K., Mundt, C., et al. (2006). Anti-inflammatory efficacy of licochalcone A: correlation of clinical potency and in vitro effects. Arch. Dermatol. Res. 298, 23-30. doi:10.1007/ s00403-006-0654-4

Koudokpon, H., Armstrong, N., Dougnon, T., Fah, L., Hounsa, E., Bankolé, H., et al. (2018). Antibacterial activity of chalcone and dihydrochalcone compounds from uvaria chamae roots against multidrug-resistant bacteria. Biomed Res. Int. 2018, 1453173. doi:10.1155/2018/1453173

Kucerova-Chlupacova, M., Kunes, J., Buchta, V., Vejsova, M., and Opletalova, V. (2015). Novel pyrazine analogs of chalcones: synthesis and evaluation of their antifungal and antimycobacterial activity. Molecules 20, 1104-1117. doi:10. 3390/molecules20011104

Kulkarni, R. R., Tupe, S. G., Gample, S. P., Chandgude, M. G., Sarkar, D., Deshpande, M. V., et al. (2014). Antifungal dimeric chalcone derivative kamalachalcone E from mallotus philippinensis. Nat. Prod. Res. 28, 245-250. doi:10.1080/14786419.2013.843178

Kumar, S., Sharma, A., Madan, B., Singhal, V., and Ghosh, B. (2007). Isoliquiritigenin inhibits IкB kinase activity and ROS generation to block TNF- $\alpha$ induced expression of cell adhesion molecules on human endothelial cells. Biochem. Pharmacol. 73, 1602-1612. doi:10.1016/j.bcp.2007.01.015

Lee, M. H., Kim, J. Y., and Ryu, J. H. (2005). Prenylflavones from psoralea corylifolia inhibit nitric oxide synthase expression through the inhibition of I-kappaB-alpha degradation in activated microglial cells. Biol. Pharm. Bull. 28, 2253-2257. doi:10.1248/bpb.28.2253

Li, R., Kenyon, G. L., Cohen, F. E., Chen, X., Gong, B., Dominguez, J. N., et al. (1995). In vitro antimalarial activity of chalcones and their derivatives. J. Med. Chem. 38, 5031-5037. doi:10.1021/jm00026a010

Liaras, K., Geronikaki, A., Glamočlija, J., Cirić, A., and Soković, M. (2011). Thiazole-based chalcones as potent antimicrobial agents. synthesis and biological evaluation. Bioorg. Med. Chem. 19, 3135-3140. doi:10.1016/j.bmc. 2011.04.007

Lichota, A., Gwozdzinski, L., and Gwozdzinski, K. (2019). Therapeutic potential of natural compounds in inflammation and chronic venous insufficiency. Eur. J. Med. Chem. 176, 68-91. doi:10.1016/j.ejmech.2019.04.075
Lima, T. C., Souza, R. J., Santos, A. D., Moraes, M. H., Biondo, N. E., Barison, A., et al. (2016). Evaluation of leishmanicidal and trypanocidal activities of phenolic compounds from calea uniflora less. Nat. Prod. Res. 30, 551-557. doi:10.1080/14786419.2015.1030740

Liu, M., Wilairat, P., Croft, S. L., Tan, A. L.-C., and Go, M.-L. (2003). Structure-activity relationships of antileishmanial and antimalarial chalcones. Bioorg. Med. Chem. 11, 2729-2738. doi:10.1016/s0968-0896(03) 00233-5

Liu, M., Yin, H., Liu, G., Dong, J., Qian, Z., and Miao, J. (2014). Xanthohumol, a prenylated chalcone from beer hops, acts as an $\alpha$-glucosidase inhibitor in vitro. J. Agric. Food Chem. 62, 5548-5554. doi:10.1021/jf500426z

Lopez, S. N., Castelli, M. V., Zacchino, S. A., Domínguez, J. N., Lobo, G., CharrisCharris, J., et al. (2001). In vitro antifungal evaluation and structure-activity relationships of a new series of chalcone derivatives and synthetic analogues, with inhibitory properties against polymers of the fungal cell wall. Bioorg. Med. Chem. 9, 1999-2013. doi:10.1016/s0968-0896(01)00116-x

Maccari, R., and Ottanà, R. (2015). Targeting aldose reductase for the treatment of diabetes complications and inflammatory diseases: new insights and future directions. J. Med. Chem. 58, 2047-2067. doi:10.1021/jm500907a

Mahapatra, D. K., Bharti, S. K., and Asati, V. (2017a). Chalcone derivatives: antiinflammatory potential and molecular targets perspectives. Curr. Top. Med. Chem. 17, 3146-3169. doi:10.2174/1568026617666170914160446

Mahapatra, D. K., Chhajed, S. S., and Shivhare, R. S. (2017b). Development of Murrayanine-Chalcone hybrids: an effort to combine two privilege scaffolds for enhancing hypoglycemic activity. Int. J. Pharm. Chem. Anal. 4, 30-34.

Mellado, M., Espinoza, L., Madrid, A., Mella, J., Chávez-Weisser, E., Diaz, K., et al. (2019). Design, synthesis, antifungal activity, and structure-activity relationship studies of chalcones and hybrid dihydrochromane-chalcones. Mol. Divers. 24 (3), 603-615. doi:10.1007/s11030-019-09967-y

Mititelu, R. R., Pădureanu, R., Băcănoiu, M., Pădureanu, V., Docea, A. O., Calina, D., et al. (2020). Inflammatory and oxidative stress markers-mirror tools in rheumatoid arthritis. Biomedicines 8, 125. doi:10.3390/biomedicines 8050125

Mocan, A., Vlase, L., Vodnar, D. C., Bischin, C., Hanganu, D., Gheldiu, A.-M., et al. (2014). Polyphenolic content, antioxidant and antimicrobial activities of Lycium barbarum L. and Lycium chinense Mill. leaves. Molecules 19, 10056-10073. doi:10.3390/molecules 190710056

Monisha, E., Suganya, V., Anuradha, V., and Syed Ali, M. (2018). Antioxidant, anti-inflammatory and antidiabetic activity of some novel chalcone and piperidine derivatives. Int. Res. J. Pharmacy Med. Sci. 2, 2581-3277.

Moosavi, M. A., Haghi, A., Rahmati, M., Taniguchi, H., Mocan, A., Echeverría, J., et al. (2018). Phytochemicals as potent modulators of autophagy for cancer therapy. Cancer Lett. 424, 46-69. doi:10.1016/j.canlet.2018.02.030

Mounika, K. L. S. (2015). Silico evaluation of alpha glucosidase and alpha amylase, inhibitory activity of chemical constituents from psoralea corylifolia. Int. J. Chem. Res. 8, 532-538.

Naidu, M. A., and Prasad, Y. R. (2018). Evaluation of antidiabetic activity of novel diarylsulfonylureachalcone hybrids in streptozotocin-induced diabetic models in rats. Asian J. Pharm. 12, S382-S405. doi:10.22377/ajp.v12i01.2464

Najafian, M., Ebrahim-Habibi, A., Yaghmaei, P., Parivar, K., and Larijani, B. (2010). Core structure of flavonoids precursor as an antihyperglycemic and antihyperlipidemic agent: an in vivo study in rats. Acta Biochim. Pol. 57, 553-560. doi:10.18388/abp.2010_2443

Ngameni, B., Watchueng, J., Boyom, F. F., Keumedjio, F., Ngadjui, B. T., Gut, J., et al. (2007). Antimalarial prenylated chalcones from the twigs of dorstenia barteri var. subtriangularis. Arkivoc 13, 116-123. doi:10.3998/ark.5550190. 0008.d14

Nowakowska, Z. (2007). A review of anti-infective and anti-inflammatory chalcones. Eur. J. Med. Chem. 42, 125-137. doi:10.1016/j.ejmech.2006. 09.019

Nyandoro, S., Nkunya, M., Josepha, C., Odalo, J., and Sattler, I. (2012). New glucopyranosylglyceryl-N-octenyl adipate and bioactivity of retro and condensed chalcones from Toussaintia orientalis. Tanzan. J. Sci. 38, 108-126.

Oh, Y. J., and Seo, Y. H. (2017). A novel chalcone-based molecule, BDP inhibits MDA-MB-231 triple-negative breast cancer cell growth by suppressing Hsp90 function. Oncol. Rep. 38, 2343-2350. doi:10.3892/or.2017.5925

Ohta, M., Fujinami, A., Kobayashi, N., Amano, A., Ishigami, A., Tokuda, H., et al. (2015). Two chalcones, 4-hydroxyderricin and xanthoangelol, stimulate 
GLUT4-dependent glucose uptake through the LKB1/AMP-activated protein kinase signaling pathway in 3T3-L1 adipocytes. Nutr. Res. 35, 618-625. doi:10. 1016/j.nutres.2015.05.010

Okuda-Tanino, A., Sugawara, D., Tashiro, T., Iwashita, M., Obara, Y., Moriya, T., et al. (2017). Licochalcones extracted from Glycyrrhiza inflata inhibit platelet aggregation accompanied by inhibition of COX-1 activity. PLoS One 12, e0173628. doi:10.1371/journal.pone.0173628

Orlikova, B., Schnekenburger, M., Zloh, M., Golais, F., Diederich, M., and Tasdemir, D. (2012). Natural chalcones as dual inhibitors of HDACs and NF-кB. Oncol. Rep. 28, 797-805. doi:10.3892/or.2012.1870

Otero, E., Vergara, S., Robledo, S. M., Cardona, W., Carda, M., Vélez, I. D., et al. (2014). Synthesis, leishmanicidal and cytotoxic activity of triclosan-chalcone, triclosan-chromone and triclosan-coumarin hybrids. Molecules 19, 13251-13266. doi:10.3390/molecules190913251

Özdemir, A., Altıntop, M. D., Turan-Zitouni, G., Çiftçi, G. A., Ertorun, I., Alataş, Ö., et al. (2015). Synthesis and evaluation of new indole-based chalcones as potential antiinflammatory agents. Eur. J. Med. Chem. 89, 304-309. doi:10. 1016/j.ejmech.2014.10.056

Padureanu, R., Albu, C. V., Mititelu, R. R., Bacanoiu, M. V., Docea, A. O., Calina, D., et al. (2019). Oxidative stress and inflammation interdependence in multiple sclerosis. J. Clin. Med. 8, 1815. doi:10.3390/jcm8111815

Pajouhesh, H., and Lenz, G. R. (2005). Medicinal chemical properties of successful central nervous system drugs. NeuroRx 2, 541-553. doi:10.1602/neurorx.2.4. 541

Passalacqua, T. G., Torres, F. A., Nogueira, C. T., De Almeida, L., Del Cistia, M. L., Dos Santos, M. B., et al. (2015). The 2', 4' -dihydroxychalcone could be explored to develop new inhibitors against the glycerol-3-phosphate dehydrogenase from leishmania species. Bioorg. Med. Chem. Lett. 25, 3564-3568. doi:10. 1016/j.bmcl.2015.06.085

Peralta, G. A., Gardoqui, J. A., Macías, F. L., Ceja, V. N., Cisneros, S. M., and Macías, C. M. (2007). Clinical and capillaroscopic evaluation in the treatment of chronic venous insufficiency with Ruscus aculeatus, hesperidin methylchalcone and ascorbic acid in venous insufficiency treatment of ambulatory patients. Int. Angiol. 26, 378.

Prasath, R., Bhavana, P., Ng, S. W., and Tiekink, E. R. (2013). The facile and efficient ultrasound-assisted synthesis of new quinoline-appended ferrocenyl chalcones and their properties. J. Organomet. Chem. 726, 62-70. doi:10.1016/j. jorganchem.2012.12.022

Purnima, S., Beena, P., Mini, R., and Swaminathan, P. (2012). Study of the effect of Adhatoda zeylanica and some related synthesized chalcones on glucose diffusion in vitro. ARPB 2, 259-263.

Rahaman, S., Prasad, Y. R., Bhuvaneswari, K., and Kumar, P. (2010). Synthesis and antihistaminic activity of novel pyrazoline derivatives. Int. J. Chem. Tech. Res. 2, $16-20$.

Raju, D. B., Rao, A. V., and Prasad, Y. R. (2018). Hybrid sulfonylurea-linked chalconoids as antidiabetic agents: evaluation of antihyperglycemic effects in streptozotocin-induced type 2 diabetic rats. Rasayan J. Chem. 11, 1334-1338. doi:10.31788/rjc.2018.1133084

Rao, M. V., Rao, A. V., and Mujavar, E. (2014). Evaluation of the in vivo hypoglycemic effect of sulfonylurea-chalcone hybrid molecules in normoglycemic rabbits. J. Global Trends Pharm. Sci. 5, 1797-1803.

Rawat, P., Kumar, M., Rahuja, N., Srivastava, D. S. L., Srivastava, A. K., and Maurya, R. (2011). Synthesis and antihyperglycemic activity of phenolic C-glycosides. Bioorg. Med. Chem. Lett 21, 228-233. doi:10.1016/j.bmcl.2010. 11.031

Real, L. (1967). Duvud C and Francois B. J. Pharmacol. Sci. 2, 37.

Ribnicky, D. M., Kuhn, P., Poulev, A., Logendra, S., Zuberi, A., Cefalu, W. T., et al. (2009). Improved absorption and bioactivity of active compounds from an antidiabetic extract of artemisia dracunculus L. Int. J. Pharm. 370, 87-92. doi:10. 1016/j.ijpharm.2008.11.012

Rocha, S., Sousa, A., Ribeiro, D., Correia, C. M., Silva, V. L., Santos, C. M., et al. (2019). A study towards drug discovery for the management of type 2 diabetes mellitus through inhibition of the carbohydrate-hydrolyzing enzymes $\alpha$-amylase and $\alpha$-glucosidase by chalcone derivatives. Food Funct. 10, 5510-5520. doi:10.1039/c9fo90045d

Rojas, J., Domínguez, J. N., Charris, J. E., Lobo, G., Payá, M., and Ferrándiz, M. L. (2002). Synthesis and inhibitory activity of dimethylamino-chalcone derivatives on the induction of nitric oxide synthase. Eur. J. Med. Chem. 37, 699-705. doi:10.1016/s0223-5234(02)01387-9

Rojas, J., Payá, M., Devesa, I., Dominguez, J. N., and Ferrándiz, M. L. (2003a). Therapeutic administration of 3, 4, 5-trimethoxy-4'-fluorochalcone, a selective inhibitor of iNOS expression, attenuates the development of adjuvant-induced arthritis in rats. Naunyn Schmiedebergs Arch. Pharmacol. 368, 225-233. doi:10. 1007/s00210-003-0780-x

Rojas, J., Payá, M., Domínguez, J. N., and Ferrándiz, M. L. (2003b). ttCH, a selective inhibitor of inducible nitric oxide synthase expression with antiarthritic properties. Eur. J. Pharmacol. 465, 183-189. doi:10.1016/s00142999(03)01457-2

Romagnoli, R., Baraldi, P. G., Carrion, M. D., Cara, C. L., Cruz-Lopez, O., Preti, D., et al. (2008). Design, synthesis, and biological evaluation of thiophene analogues of chalcones. Bioorg. Med. Chem. 16, 5367-5376. doi:10.1016/j. bmc.2008.04.026

Ryu, H. W., Lee, B. W., Curtis-Long, M. J., Jung, S., Ryu, Y. B., Lee, W. S., et al. (2010). Polyphenols from Broussonetia papyrifera displaying potent a-glucosidase inhibition. J. Agric. Food Chem. 58, 202-208. doi:10.1021/ jf903068k

Salehi, B., Capanoglu, E., Adrar, N., Catalkaya, G., Shaheen, S., Jaffer, M., et al. (2019a). Cucurbits plants: a key emphasis to its pharmacological potential. Molecules 24, 1854. doi:10.3390/molecules24101854

Salehi, B., Lopez-Jornet, P., Pons-Fuster López, E., Calina, D., Sharifi-Rad, M., Ramírez-Alarcón, K., et al. (2019b). Plant-derived bioactives in oral mucosal lesions: a key emphasis to curcumin, lycopene, chamomile, aloe vera, green tea and coffee properties. Biomolecules 9, 106. doi:10.3390/ biom 9030106

Salehi, B., Sestito, S., Rapposelli, S., Peron, G., Calina, D., Sharifi-Rad, M., et al. (2019c). Epibatidine: a promising natural alkaloid in health. Biomolecules 9, 6 doi:10.3390/biom 9010006

Salehi, B., Sharifi-Rad, J., Capanoglu, E., Adrar, N., Catalkaya, G., Shaheen, S., et al. (2019d). Cucurbita plants: from farm to industry. Appl. Sci. 9, 3387. doi:10. 3390/app9163387

Salehi, B., Shivaprasad Shetty, M., Anil Kumar, V. N., Živković, J., Calina, D., et al. (2019e). Veronica plants-drifting from farm to traditional healing, food application, and phytopharmacology. Molecules 24, 2454. doi:10.3390/ molecules 24132454

Salehi, B., Calina, D., Docea, A. O., Koirala, N., Aryal, S., Lombardo, D., et al. (2020a). Curcumin's nanomedicine formulations for therapeutic application in neurological diseases. J. Clin. Med. 9, 430. doi:10.3390/jcm9020430

Salehi, B., Rescigno, A., Dettori, T., Calina, D., Docea, A. O., Singh, L., et al. (2020b). Avocado-Soybean unsaponifiables: a panoply of potentialities to Be exploited. Biomolecules 10, 130. doi:10.3390/biom10010130

Salehi, B., Sharifi-Rad, J., Cappellini, F., Reiner, Ž., Zorzan, D., Imran, M., et al. (2020c). The therapeutic potential of anthocyanins: current approaches based on their molecular mechanism of action. Front. Pharmacol. 11, 1300. doi:10. 3389/fphar.2020.01300

Salem, M. M., and Werbovetz, K. A. (2005). Antiprotozoal compounds from Psorothamnus p olydenius. J. Nat. Prod. 68, 108-111. doi:10.1021/np049682k

Salem, M. M., and Werbovetz, K. A. (2006). Isoflavonoids and other compounds from Psorothamnus a rborescens with antiprotozoal activities. J. Nat. Prod. 69, 43-49. doi:10.1021/np049682k

Satyanarayana, M., Tiwari, P., Tripathi, B. K., Srivastava, A., and Pratap, R. (2004). Synthesis and antihyperglycemic activity of chalcone based aryloxypropanolamines. Bioorg. Med. Chem. 12, 883-889. doi:10.1016/j.bmc.2003.12.026

Scheau, C., Badarau, I. A., Mihai, L.-G., Scheau, A.-E., Costache, D. O., Constantin, C., et al. (2020). Cannabinoids in the pathophysiology of skin inflammation. Molecules 25, 652. doi:10.3390/molecules25030652

Schroder, J. (1999). The chalcone/stibene synthase-type family of condensing enzymes. Compr. Nat. Prod. Chem. 1, 749-771. doi:10.1016/b978-0-08091283-7.00029-1

Schweiger, D., Baufeld, C., Drescher, P., Oltrogge, B., Höpfner, S., Mess, A., et al. (2013). Efficacy of a new tonic containing urea, lactate, polidocanol, and glycyrrhiza inflata root extract in the treatment of a dry, itchy, and subclinically inflamed scalp. Skin Pharmacol. Physiol. 26, 108-118. doi:10.1159/000348473

Semwal, D. K., Rawat, U., Semwal, R., Singh, R., Krishan, P., Singh, M., et al. (2009). Chemical constituents from the leaves of Boehmeria rugulosa with antidiabetic 
and antimicrobial activities. J. Asian Nat. Prod. Res. 11, 1045-1055. doi:10.1080/ 10286020903352526

Sengupta, S. A., Maity, T. K., and Samanta, S. (2017). Synthesis, biological screening and in silico studies of chalcone based novel phenyl urea derivatives as potential antihyperglycemics. J. Pharm. Res. 16, 237-246. doi:10.18579/jpcrkc/2017/16/3/118765

Seo, W. D., Kim, J. H., Kang, J. E., Ryu, H. W., Curtis-Long, M. J., Lee, H. S., et al. (2005). Sulfonamide chalcone as a new class of a-glucosidase inhibitors. Bioorg. Med. Chem. Lett. 15, 5514-5516. doi:10.1016/j.bmcl.2005.08.087

Sharifi-Rad, J., Kamiloglu, S., Yeskaliyeva, B., Beyatli, A., Alfred, M. A., Salehi, B., et al. (2020a). Pharmacological activities of Psoralidin: a comprehensive review of the molecular mechanisms of action. Front. Pharmacol. 11, 571459. doi:10. 3389/fphar.2020.571459

Sharifi-Rad, J., Rodrigues, C. F., Sharopov, F., Docea, A. O., Can Karaca, A., Sharifi-Rad, M., et al. (2020b). Diet, lifestyle and cardiovascular diseases: linking pathophysiology to cardioprotective effects of natural bioactive compounds. Int. J. Environ. Res. Public Health 17, 2326. doi:10.3390/ ijerph17072326

Sharifi-Rad, M., Anil Kumar, N. V., Zucca, P., Varoni, E. M., Dini, L., Panzarini, E., et al. (2020c). Lifestyle, oxidative stress, and antioxidants: back and Forth in the pathophysiology of chronic diseases. Front. Physiol. 11, 694. doi:10.3389/fphys. 2020.00694

Sharifi-Rad, M., Lankatillake, C., Dias, D. A., Docea, A. O., Mahomoodally, M. F., Lobine, D., et al. (2020d). Impact of natural compounds on neurodegenerative disorders: from preclinical to pharmacotherapeutics. J. Clin. Med. 9, 1061. doi: $10.3390 / j \mathrm{~cm} 9041061$

Sharifi-Rad, M., Lankatillake, C., Dias, D. A., Docea, A. O., Mahomoodally, M. F., Lobine, D., et al. (2020e). Impact of natural compounds on neurodegenerative disorders: from preclinical to pharmacotherapeutics. J. Clin. Med. 9, 1061. doi:10.3390/jcm9041061

Shibata, S. (1994). Anti-tumorigenic chalcones. Stem cells 12, 44-52. doi:10.1002/ stem. 5530120109

Shimokoriyama, M. (1962). Flavanones, chalcones and aurones. In: T. Geissman (ed.) The Chemistry of Flavonoid Compounds. New York: The Macmillan Co.

Shin, J., Jang, M. G., Park, J. C., Do Koo, Y., Lee, J. Y., Park, K. S., et al. (2018). Antidiabetic effects of trihydroxychalcone derivatives via activation of AMPactivated protein kinase. J. Ind. Eng. Chem. 60, 177-184. doi:10.1016/j.jiec.2017. 11.003

Shirley, B. W. (1996). Flavonoid biosynthesis:'new'functions for an 'old'pathway. Trends Plant Sci. 1, 377-382.

Shukla, P., Satyanarayana, M., Verma, P. C., Tiwari, J., Dwivedi, A. P., Srivastava, R., et al. (2017). Chalcone-based aryloxypropanolamine as a potential antidiabetic and antidyslipidaemic agent. Curr. Sci. 112, 1675-1689. doi:10. $18520 / \mathrm{cs} / \mathrm{v} 112 / \mathrm{i} 08 / 1675-1689$

Shukla, P., Singh, A. B., Srivastava, A. K., and Pratap, R. (2007). Chalcone based aryloxypropanolamines as potential antihyperglycemic agents. Bioorg. Med. Chem. Lett. 17, 799-802. doi:10.1016/j.bmcl.2006.10.068

Siddiqui, Z. N., Praveen, S., Musthafa, T. M., Ahmad, A., and Khan, A. U. (2012). Thermal solvent-free synthesis of chromonyl chalcones, pyrazolines and their in vitro antibacterial, antifungal activities. J. Enzym. Inhib. Med. Chem. 27, 84-91. doi:10.3109/14756366.2011.577035

Sifaki, M., Calina, D., Docea, A. O., Tsioumas, S., Katsarou, M. S., Papadogiorgaki, S., et al. (2020). A novel approach regarding the anti-aging of facial skin through collagen reorganization. Exp. Ther. Med. 19, 717-721. doi:10.3892/etm.2019. 8254

Singh, A., Fong, G., Liu, J., Wu, Y.-H., Chang, K., Park, W., et al. (2018). Synthesis and preliminary antimicrobial analysis of isatin-ferrocene and isatin-ferrocenyl chalcone conjugates. ACS Omega 3, 5808-5813. doi:10. 1021/acsomega.8b00553

Sousa-Batista, A. D. J., Philipon, C. I. S., De Souza Albernaz, M., Pinto, S. R., RossiBergmann, B., and Santos-Oliveira, R. (2018). New chalcone compound as a promising antileishmanial drug for an old neglected disease: biological evaluation using radiolabelled biodistribution. J. Glob. Antimicrob. Resist. 13, 139-142. doi:10.1016/j.jgar.2017.11.012

Sulzberger, M., Worthmann, A. C., Holtzmann, U., Buck, B., Jung, K., Schoelermann, A., et al. (2016). Effective treatment for sensitive skin: 4-tbutylcyclohexanol and licochalcone A. J. Eur. Acad. Dermatol. Venereol. 30, 9-17. doi:10.1111/jdv.13529
Sun, H., Li, Y., Zhang, X., Lei, Y., Ding, W., Zhao, X., et al. (2015). Synthesis, a-glucosidase inhibitory and molecular docking studies of prenylated and geranylated flavones, isoflavones and chalcones. Bioorg. Med. Chem. Lett. 25, 4567-4571. doi:10.1016/j.bmcl.2015.08.059

Sun, H., Wang, D., Song, X., Zhang, Y., Ding, W., Peng, X., et al. (2017). Natural prenylchalconaringenins and prenylnaringenins as antidiabetic agents: a-glucosidase and $a$-amylase inhibition and in vivo antihyperglycemic and antihyperlipidemic effects. J. Agric. Food Chem. 65, 1574-1581. doi:10.1021/acs. jafc.6b05445

Svetaz, L., Tapia, A., López, S. N., Furlán, R. L., Petenatti, E., Pioli, R., et al. (2004). Antifungal chalcones and new caffeic acid esters from Zuccagnia punctata acting against soybean infecting fungi. J. Agric. Food Chem. 52, 3297-3300. doi:10.1021/jf035213x

Syam, S., Abdelwahab, S. I., Al-Mamary, M. A., and Mohan, S. (2012). Synthesis of chalcones with anticancer activities. Molecules 17, 6179-6195. doi:10.3390/ molecules 17066179

Tajammal, A., Batool, M., Ramzan, A., Samra, M. M., Mahnoor, I., Verpoort, F., et al. (2017). Synthesis, antihyperglycemic activity and computational studies of antioxidant chalcones and flavanones derived from 2, 5 dihydroxyacetophenone. J. Mol. Struct. 1148, 512-520. doi:10.1016/j. molstruc.2017.07.042

Tajuddeen, N., Isah, M. B., Suleiman, M. A., Van Heerden, F. R., and Ibrahim, M. A. (2018). The chemotherapeutic potential of chalcones against leishmaniases: a review. Int. J. Antimicrob. Agents 51, 311-318. doi:10.1016/j.ijantimicag.2017.06.010

Takahashi, M., Maeda, S., Ogura, K., Terano, A., and Omata, M. (1998). The possible role of vascular endothelial growth factor (VEGF) in gastric ulcer healing: effect of sofalcone on VEGF release in vitro. J. Clin. Gastroenterol. 27, S178-S182. doi:10.1097/00004836-199800001-00029

Tan, R. X., Meng, J. C., and Hostettmann, K. (2000). Phytochemical investigation of some traditional chinese medicines and endophyte cultures. Pharm. Biol. 38, 25-32. doi:10.1076/phbi.38.6.25.5955

Tang, C., Zhu, L., Chen, Y., Qin, R., Mei, Z., Xu, J., et al. (2014). Synthesis and biological evaluation of oleanolic acid derivative-chalcone conjugates as a-glucosidase inhibitors. RSC Adv. 4, 10862-10874. doi:10.1039/ c3ra46492j

Toiu, A., Mocan, A., Vlase, L., Pârvu, A. E., Vodnar, D. C., Gheldiu, A.-M., et al. (2019). Comparative phytochemical profile, antioxidant, antimicrobial and in vivo anti-inflammatory activity of different extracts of traditionally used romanian ajuga genevensis L. and A. reptans L. (Lamiaceae). Molecules 24, 1597. doi:10.3390/molecules 24081597

Tomar, V., Bhattacharjee, G., and Kumar, A. (2007). Synthesis and antimicrobial evaluation of new chalcones containing piperazine or 2, 5-dichlorothiophene moiety. Bioorg. Med. Chem. Lett. 17, 5321-5324. doi:10.1016/j.bmcl.2007.08. 021

Tomar, V., Bhattacharjee, G., Rajakumar, S., Srivastava, K., and Puri, S. (2010). Synthesis of new chalcone derivatives containing acridinyl moiety with potential antimalarial activity. Eur. J. Med. Chem. 45, 745-751. doi:10.1016/ j.ejmech.2009.11.022

Tomás-Barberán, F. A., and Clifford, M. N. (2000). Flavanones, chalcones and dihydrochalcones-nature, occurrence and dietary burden. J. Sci. Food Agric. 80, 1073-1080. doi:10.1002/(sici)1097-0010(20000515)80:7<1073::aid-jsfa568 $>3$. $0 . \mathrm{co} ; 2-\mathrm{b}$

Torres-Santos, E. C., Moreira, D. L., Kaplan, M. a. C., Meirelles, M. N., and RossiBergmann, B. (1999). Selective effect of $2^{\prime}, 6^{\prime}$-dihydroxy-4'-methoxychalcone isolated from Piper aduncum on Leishmania amazonensis. Antimicrob. Agents Chemother. 43, 1234-1241. doi:10.1128/AAC.43.5.1234

Tsatsakis, A.Docea, A. O., Calina, D., Tsarouhas, K., Zamfira, L.-M., Mitrut, R., et al. (2019). A mechanistic and pathophysiological approach for stroke associated with drugs of abuse. J. Clin. Med. 8, 1295. doi:10.3390/ jcm8091295

Tsatsakis, A. M.Docea, A. O., Calina, D., Buga, A. M., Zlatian, O., Gutnikov, S., et al. (2019). Hormetic Neurobehavioral effects of low dose toxic chemical mixtures in real-life risk simulation (RLRS) in rats. Food Chem. Toxicol. 125, 141-149. doi:10.1016/j.fct.2018.12.043

Udompataikul, M., and Srisatwaja, W. (2011). Comparative trial of moisturizer containing licochalcone A vs. hydrocortisone lotion in the treatment of childhood atopic dermatitis: a pilot study. J. Eur. Acad. Dermatol. Venereol. 25, 660-665. doi:10.1111/j.1468-3083.2010.03845.x 
Ugwu, D. I., Ezema, B. E., Okoro, U. C., Eze, F. U., Ekoh, O. C., Egbujor, M. C., et al. (2015). Syntheses and pharmacological applications of chalcones: a review. Int. J. Chem. Sci. 13, 459-500.

Ungureanu, A., Zlatian, O., Mitroi, G., Drocaş, A., Ţîrcă, T., Călina, D., et al. (2017). Staphylococcus aureus colonisation in patients from a primary regional hospital. Mol. Med. Rep. 16, 8771-8780. doi:10.3892/mmr.2017.7746

Vijaya Bhaskar Reddy, M., Hung, H.-Y., Kuo, P.-C., Huang, G.-J., Chan, Y.-Y., Huang, S.-C., et al. (2017). Synthesis and biological evaluation of chalcone, dihydrochalcone, and 1,3-diarylpropane analogs as anti-inflammatory agents. Bioorg. Med. Chem. Lett. 27, 1547-1550. doi:10.1016/j.bmcl.2017.02.038

Viveka, S., Naik, P., and Nagaraja, G. K. (2014). Synthesis, characterization of new imidazoquinonyl chalcones and pyrazolines as potential anticancer and antioxidant agents. Med. Chem. Res. 23, 4189-4197. doi:10.1007/s00044014-0998-9

Wananukul, S., Chatproedprai, S., Chunharas, A., Limpongsanuruk, W., Singalavanija, S., Nitiyarom, R., et al. (2013). Randomized, double-blind, split-side, comparison study of moisturizer containing licochalcone A and $1 \%$ hydrocortisone in the treatment of childhood atopic dermatitis. J. Med. Assoc. Thai. 96, 1135-1142.

Watanabe, Y., Nagai, Y., Honda, H., Okamoto, N., Yamamoto, S., Hamashima, T., et al. (2016). Isoliquiritigenin attenuates adipose tissue inflammation in vitro and adipose tissue fibrosis through inhibition of innate immune responses in mice. Sci. Rep. 6, 23097. doi:10.1038/srep23097

Weber, T., Ceilley, R., Buerger, A., Kolbe, L., Trookman, N., Rizer, R., et al. (2006). Skin tolerance, efficacy, and quality of life of patients with red facial skin using a skin care regimen containing licochalcone A. J. Cosmet. Dermatol. 5, 227-232. doi:10.1111/j.1473-2165.2006.00261.x

Weindorf, N., and Schultz-Ehrenburg, U. (1987). Controlled study of increasing venous tone in primary varicose veins by oral administration of Ruscus aculeatus and trimethylhespiridinchalcone. Z. Hautkr. 62, 28-38.

Won, S.-J., Liu, C.-T., Tsao, L.-T., Weng, J.-R., Ko, H.-H., Wang, J.-P., et al. (2005). Synthetic chalcones as potential anti-inflammatory and cancer chemopreventive agents. Eur. J. Med. Chem. 40, 103-112. doi:10.1016/j.ejmech.2004.09.006

Yadav, N., Dixit, S. K., Bhattacharya, A., Mishra, L. C., Sharma, M., Awasthi, S. K., et al. (2012). Antimalarial activity of newly synthesized chalcone derivatives in vitro. Chem. Biol. Drug Des. 80, 340-347. doi:10.1111/j.1747-0285.2012.01383.x

Yin, B.-T., Yan, C.-Y., Peng, X.-M., Zhang, S.-L., Rasheed, S., Geng, R.-X., et al. (2014). Synthesis and biological evaluation of $\alpha$-triazolyl chalcones as a new type of potential antimicrobial agents and their interaction with calf thymus DNA and human serum albumin. Eur. J. Med. Chem. 71, 148-159. doi:10.1016/ j.ejmech.2013.11.003

Zajac, M., Muszalska, I., and Jelinska, A. (2016). New molecular targets of anticancer therapy-current status and perspectives. Curr. Med. Chem. 23, 4176-4220. doi:10.2174/0929867323666160814002150

Zhang, L., and Lu, Y. (2012). Inhibitory activities of extracts from Cleistocalyx operculatus flower buds on pancreatic lipase and a-amylase. Eur. Food Res. Technol. 235, 1133-1139. doi:10.1248/bpb.26.61

Zhao, F., Nozawa, H., Daikonnya, A., Kondo, K., and Kitanaka, S. (2003). Inhibitors of nitric oxide production from hops (Humulus lupulus L.). Biol. Pharm. Bull. 26, 61-65. doi:10.1248/bpb.26.61

Zhu, P., Huang, W., Li, J., Ma, X., Hu, M., Wang, Y., et al. (2018). Design, synthesis chalcone derivatives as AdipoR agonist for type 2 diabetes. Chem. Biol. Drug Des. 92, 1525-1536. doi:10.1111/cbdd.13319

Zhuang, C., Zhang, W., Sheng, C., Zhang, W., Xing, C., and Miao, Z. (2017). Chalcone: a privileged structure in medicinal chemistry. Chem. Rev. 117, 7762-7810. doi:10.1021/acs.chemrev.7b00020

Žiberna, L., Šamec, D., Mocan, A., Nabavi, S. F., Bishayee, A., Farooqi, A. A., et al. (2017). Oleanolic acid alters multiple cell signaling pathways: implication in cancer prevention and therapy. Int. J. Mol. Sci. 18, 643. doi:10.3390/ ijms 18030643

Zuzarte, M., Vale-Silva, L., Gonçalves, M., Cavaleiro, C., Vaz, S., Canhoto, J., et al. (2012). Antifungal activity of phenolic-rich Lavandula multifida L. essential oil. Eur. J. Clin. Microbiol. Infect. Dis. 31, 1359-1366. doi:10.1007/s10096-011-1450-4

Conflict of Interest: The authors declare that the research was conducted in the absence of any commercial or financial relationships that could be construed as a potential conflict of interest.

Copyright (C) 2021 Salehi, Quispe, Chamkhi, El Omari, Balahbib, Sharifi-Rad, Bouyahya, Akram, Iqbal, Docea, Caruntu, Leyva-Gómez, Dey, Martorell, Calina, López and Les. This is an open-access article distributed under the terms of the Creative Commons Attribution License (CC BY). The use, distribution or reproduction in other forums is permitted, provided the original author $(s)$ and the copyright owner(s) are credited and that the original publication in this journal is cited, in accordance with accepted academic practice. No use, distribution or reproduction is permitted which does not comply with these terms. 